\title{
Density matrix study of ground state depletion towards sub-diffraction-limited spontaneous Raman scattering spectroscopy
}

Steffen Rieger, Thomas Würthwein, Kai Sparenberg, Klaus-Jochen Boller, and Carsten Fallnich

Citation: The Journal of Chemical Physics 148, 204110 (2018); doi: 10.1063/1.5009278

View online: https://doi.org/10.1063/1.5009278

View Table of Contents: http://aip.scitation.org/toc/jcp/148/20

Published by the American Institute of Physics

\section{Articles you may be interested in}

Perspective: Ring-polymer instanton theory

The Journal of Chemical Physics 148, 200901 (2018); 10.1063/1.5028352

Coupled-cluster treatment of molecular strong-field ionization

The Journal of Chemical Physics 148, 204102 (2018); 10.1063/1.5028179

Efficient calculation of beyond RPA correlation energies in the dielectric matrix formalism

The Journal of Chemical Physics 148, 204104 (2018); 10.1063/1.5025938

Three-dimensional single-molecule localization with nanometer accuracy using Metal-Induced Energy

Transfer (MIET) imaging

The Journal of Chemical Physics 148, 204201 (2018); 10.1063/1.5027074

Low-lying excited states by constrained DFT

The Journal of Chemical Physics 148, 144103 (2018); 10.1063/1.5018615

An atomic mean-field spin-orbit approach within exact two-component theory for a non-perturbative treatment of spin-orbit coupling

The Journal of Chemical Physics 148, 144108 (2018); 10.1063/1.5023750

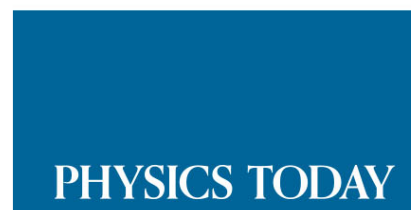

WHITEPAPERS
ADVANCED LIGHT CURE ADHESIVES

Take a closer look at what these environmentally friendly adhesive systems can do
READ NOW

PRESENTED BY

Q MASTERBOND: 


\title{
Density matrix study of ground state depletion towards sub-diffraction-limited spontaneous Raman scattering spectroscopy
}

\author{
Steffen Rieger, ${ }^{1,2, a)}$ Thomas Würthwein, ${ }^{1}$ Kai Sparenberg, ${ }^{1}$ Klaus-Jochen Boller, ${ }^{1,3}$ \\ and Carsten Fallnich ${ }^{1,2,3}$ \\ ${ }^{1}$ Institute of Applied Physics, University of Münster, Corrensstraße 2, 48149 Münster, Germany \\ ${ }^{2}$ Cells-in-Motion Cluster of Excellence (EXC 1003-CiM), Waldeyerstraße 15, 48149 Münster, Germany \\ ${ }^{3}$ MESA+ Institute for Nanotechnology, University of Twente, Enschede, The Netherlands
}

(Received 14 October 2017; accepted 30 April 2018; published online 29 May 2018)

\begin{abstract}
The suppression of Raman scattering is of high interest for the achievement of sub-diffraction-limited resolution in Raman scattering spectroscopy and microscopy. We present density matrix calculations of the suppression of spontaneous Raman scattering via ground state depletion in a level system based on the molecule tris(bipyridine)ruthenium(II). This particular molecule has been earlier used for an experimental demonstration of the suppression of spontaneous Raman scattering, allowing us to successfully verify the validity of our numerical calculations by a comparison to the experimental results. We investigate the required level of detail of the molecule model as well as the influence of certain molecule and pulse parameters on the Raman scattering suppression. It was found that pulses with a duration longer than the lifetime of the electronic states allow for a high suppression of the Raman scattering. Pulses shorter than the coherence lifetime between the ground state and electronic states lead to a similarly high suppression but also accomplish the suppression with more than one order of magnitude lower pulse energy fluence. Additionally, using a laser wavelength that is in resonance with one of the electronic transitions of the sample should allow suppressing the Raman scattering with four to six orders of magnitude lower pulse energy fluence. Published by AIP Publishing. https://doi.org/10.1063/1.5009278
\end{abstract}

\section{INTRODUCTION}

Since the development of super-resolution techniques such as stimulated emission depletion (STED) microscopy, ${ }^{1}$ stochastic optical reconstruction microscopy (STORM), ${ }^{2}$ and photoactivated localization microscopy (PALM), ${ }^{3}$ fluorescence microscopy with sub-diffraction-limited resolution has become an important tool for the investigation of biological and technical samples on the nanometer scale. Recently, the resolution limit of fluorescence microscopy was improved down to a groundbreaking $1 \mathrm{~nm}$ by the development of minimal photon flux (MINFLUX) microscopy. ${ }^{4}$ However, in labelfree Raman microscopy, a comparable spatial resolution has not yet been achieved, although such a development would be of high interest as Raman scattering provides chemically selective information about the sample without possible collateral side-effects on the cell metabolism as induced by some fluorophores.

In the field of Raman microscopy, advances have been made in the past to enhance the resolution (relative to the diffraction limit) by employing structured beams enhancing the resolution of coherent anti-Stokes Raman scattering (CARS) by about a factor of two. ${ }^{5,6} \mathrm{~A}$ more recent work on stimulated Raman scattering (SRS) demonstrated the manipulation of the vibrational coherences of a molecule with an additional donut-shaped control beam in order to promote a

a)Electronic mail: steffen.rieger@uni-muenster.de competing four-wave mixing process achieving a comparable resolution enhancement by a factor of two. ${ }^{7}$ However, an even higher resolution enhancement should be possible when using higher control pulse energies, as the employed mechanism of resolution enhancement was based on the saturable suppression of the observed signal similar to STED microscopy. A related approach has also gained attention recently, which is the suppression of SRS by depleting the pump photons with a competing Raman scattering process driven by a strong second Stokes beam. ${ }^{8}$ It is a promising method with a well-understood theoretical background, ${ }^{9}$ but with the disadvantage that it is not applicable to the fundamental process of spontaneous Raman scattering as it requires the presence of multiple (pump and Stokes) beams. In another recent work, the depletion of the ground state of a molecule by an ultraviolet (UV) pulse was used for the suppression of spontaneous Raman scattering. ${ }^{10}$ It was estimated that the observed suppression, if used in a STED-like Raman microscopy approach, should lead to a resolution enhancement by a factor of three. An even higher resolution enhancement should generally be possible using a higher pulse energy for stronger ground state depletion (GSD). For this reason and because it is a straightforward approach applicable to all variants of Raman scattering, GSD is of high interest for a potential application in resolution-enhanced microscopy.

Extensive numerical investigations have been performed in the past on the topic of spatial resolution enhancement in coherent Raman scattering microscopy by employing density 
matrix calculations of molecules irradiated with light in order to obtain information about consequent changes to the population of the molecular states and the accompanying Raman scattering processes. ${ }^{11}$ In contrast to using rate equations, density matrix calculations provide the possibility to investigate coherent population effects which are involved in the excitation of molecules with femtosecond pulses as well as coherent Raman scattering processes. In these studies, it was, e.g., proposed that the spatial resolution of a CARS setup can be enhanced by analyzing the intensity-dependent frequency of amplitude modulation sidebands, caused in the spectrum by Rabi oscillations, which can be driven by a Gaussian-shaped control beam in the mid-infrared (MIR). ${ }^{12}$ Another option for resolution enhancement would be the suppression of the CARS signal by an incoherent population of the vibrational states in a donut-shaped region around the focus to be achieved by irradiating the sample with MIR light ${ }^{13}$ or a pair of control light fields in the scheme of stimulated emission pumping (STEP). ${ }^{14}$ Similarly, a suppression of the CARS signal around the focal spot might be achievable by a depletion of the ground state of the sample's molecules. ${ }^{15}$ Also for SRS a resolution enhancement was proposed utilizing a donut-shaped control beam with a high intensity to generate a saturated Raman signal around the center of the focus of a Gaussian beam with a lower intensity. ${ }^{16}$

However, the above theoretical investigations were based on oversimplified molecular models, containing, besides the ground state of the molecule, only one or two electronic and vibrational states, respectively. By contrast, real molecules always feature a variety of both types of states. In many cases, this difference should have an impact on theoretical investigations, especially on GSD, as a higher number of states to which population can be transferred should generally result in a higher possible depletion.

Following this argument, it would be preferable to perform density matrix calculations on the basis of level systems that, in number and properties of their states, closer resemble molecules for which Raman scattering suppression was already experimentally demonstrated. Furthermore, if the parameters of the molecular transitions, i.e., frequencies, population lifetimes, coherence lifetimes, and transition dipole moments, would be close to the ones of the chosen molecule, it should also be possible to predict the pulse energy fluence required for a certain suppression of the Raman scattering and to compare the results of the calculations with existing experimental data. From there on, it would be possible to investigate the influence of a variation of molecule and pulse parameters on the Raman scattering suppression in order to identify conditions under which the efficiency of the suppression can be increased such that the proposed method can be applied to a variety of samples with different properties using low pulse energy fluences for improved biocompatibility.

Here, we report on density matrix calculations on the suppression of spontaneous Raman scattering due to GSD in a level system based on the molecule tris(bipyridine) ruthenium(II) $\left(\mathrm{Ru}(\mathrm{bpy})_{3}^{2+}\right)$, which was employed in the experimental demonstration of GSD reported in Ref. 10. There, the spontaneous Raman scattering was suppressed by up to $50 \%$ via depletion of the ground state of the molecules using nanosecond UV laser pulses at a wavelength of $355 \mathrm{~nm}$. It was estimated that the observed Raman scattering suppression would be sufficient for a resolution enhancement by a factor of three when taking difference images with a combination of Gaussian and donut-shaped beams. Using the same molecule, $\mathrm{Ru}(\mathrm{bpy})_{3}^{2+}$, as a model system for the density matrix calculations enabled the possibility to compare the experimental and numerical results in order to verify that our calculations can be used to accurately predict the physical behavior of a molecule. For the same reason, we studied the suppression of the spontaneous Raman scattering instead of CARS ${ }^{12-15}$ or $\mathrm{SRS}^{8,9,16}$ which was exclusively investigated in previous studies.

Our density matrix calculations are then used to assess under which conditions simplifications to the modeled level system, i.e., the neglection of vibrational or electronic states, as they were made in earlier studies, should be justified. The density matrix calculations are also employed to gain insight into the dependence of the spontaneous Raman scattering on several parameters such as the duration of the laser pulse, the coherence lifetimes between the molecular states, and the wavelength of the laser radiation. From these investigations, conditions are identified under which the most efficient GSD can be achieved and, thereby, a high resolution enhancement can be obtained in a potential future application.

\section{CONCEPT OF RESOLUTION-ENHANCED RAMAN SCATTERING SPECTROSCOPY VIA GROUND STATE DEPLETION}

We like to briefly recall how GSD can be applied to achieve a resolution enhancement in Raman scattering spectroscopy as proposed in Ref. 10. The key of this method is the irradiation of sample molecules with pulsed UV laser light, which generates a Raman scattering signal, while simultaneously depleting the ground state of the sample. Therefore, an increase of the pulse energy will result in an increase of the detected Raman scattering signal which saturates at high pulse energies, because population is transferred out of the ground state, from which the Raman scattering process originates.

In a potential experimental realization, a sample would be illuminated with a combination of Gaussian and donut-shaped beams [see Fig. 1(a)]. By scanning the sample orthogonally to the optical axis and detecting the Raman scattered light (e.g., in the backwards direction) at each position, a Raman scattering image can be obtained. In the case of illumination with a Gaussian beam, this would be a diffraction-limited image of conventional resolution [see Fig. 1(b)].

A resolution-enhanced image [see Fig. 1(c)] can be obtained from two consecutive imaging processes using a donut-shaped beam as well as a beam that is a combination of the donut-shaped beam together with a Gaussian beam of (e.g., ten-fold) lower intensity. The intensity of the donutshaped beam would be chosen such that it would drive the Raman scattering signal into saturation in its regions of highest intensity. Therefore, the addition of the Gaussian beam would - due to the saturation-only insignificantly increase the Raman scattering emitted from these regions. A subtraction of the images obtained by scanning the sample with the two differently structured beams would, therefore, result in an 
(a) Setup

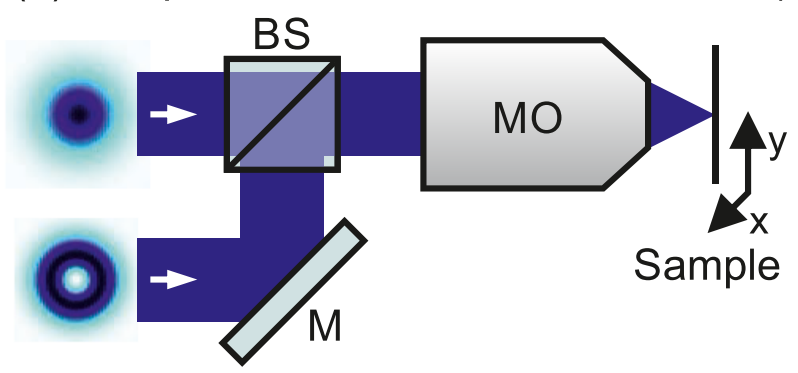

(c) Generation of resolution-enhanced image
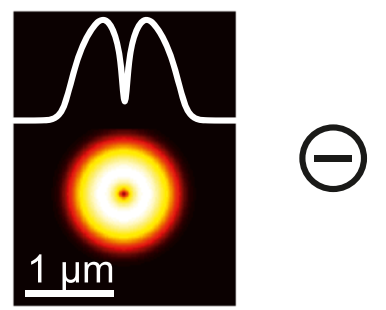

(b) Conventional image
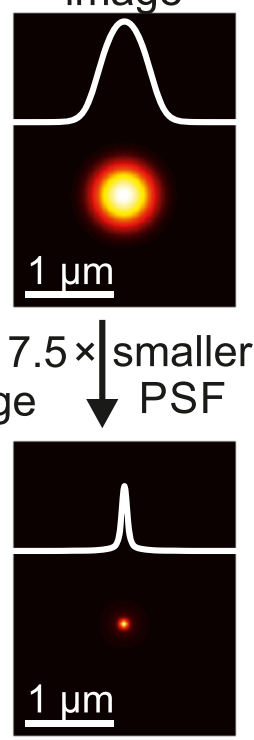

FIG. 1. Principle of resolutionenhanced Raman scattering spectroscopy via ground state depletion as introduced in Ref. 10. (a) UV Raman scattering setup using a combination of donut-shaped and Gaussian beams. BS: beam splitter; M: mirror; MO: microscope objective. (b) Conventional diffraction-limited image obtained by scanning a scattering center with a Gaussian shaped beam [normalized independently from the images shown in (c)]. (c) Generation of a resolution-enhanced image [smaller point spread function (PSF)] by subtracting an image obtained with a donut-shaped beam from the one obtained with a combination of donut-shaped and Gaussian beams. All images are displayed as false color $2 \mathrm{D}$ representation and horizontal cross section. image, in which mainly the Raman scattering signal from the center of the Gaussian beam remains. This reconstruction provides a significant resolution enhancement (here by a factor of 7.5) in comparison to a conventional image obtained with a Gaussian beam [Fig. 1(b)].

\section{DENSITY MATRIX MODEL OF Ru(bpy) ${ }_{3}^{2+}$}

In order to numerically study the spontaneous Raman scattering suppression in $\mathrm{Ru}(\mathrm{bpy})_{3}^{2+}$, a level system based on its molecular states was used for the calculations (see Fig. 2). Therefore, information about the properties of all states

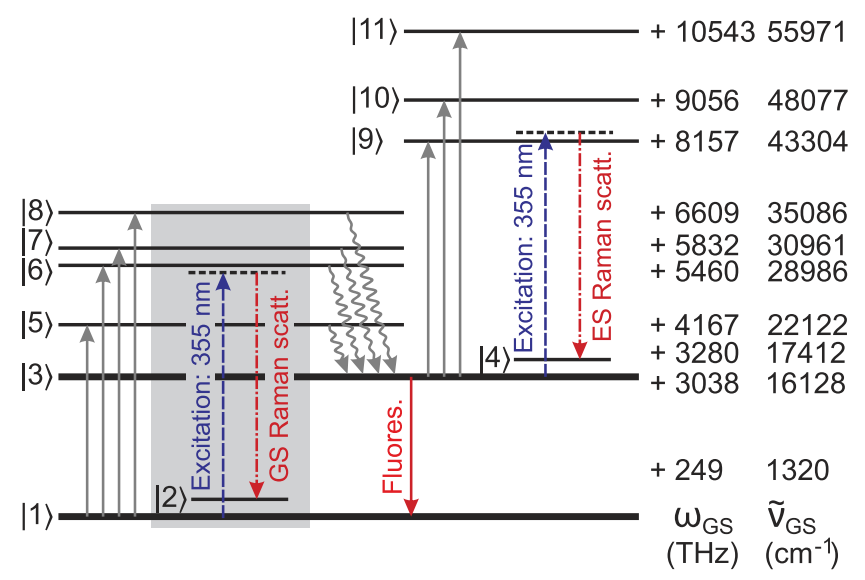

FIG. 2. Jablonski diagram of the states included in the density matrix calculations based on the molecule tris(bipyridine)ruthenium(II). Dashed blue, dashed-dotted red, and solid red arrows represent the optical frequencies of the incident laser light and Raman scattering or fluorescence as denoted. Straight gray arrows indicate transitions between states as indicated in the text. Wavy gray arrows indicate the non-radiative relaxation processes responsible for populating the lowest excited state. Dashed black lines represent the virtual states induced by the laser light during ground state (GS) and excited state (ES) Raman scattering. A gray box highlights the ground state Raman scattering process that is suppressed by the depletion of the ground state. All states (except vibrational states) are drawn to scale to their respective frequency difference. Frequency values are given in units of angular frequency $\omega(\mathrm{THz})$ as well as wavenumber $\tilde{v}\left(\mathrm{~cm}^{-1}\right)$. Detailed information about the individual states can be found in Table I (Appendix A 3). included in the level system, i.e., their frequencies and lifetimes as well as transition dipole moments and coherence lifetimes between pairs of states, was collected from independent measurements and the literature. ${ }^{17-23}$

The specific molecular states and transitions of $\mathrm{Ru}(\mathrm{bpy})_{3}^{2+}$ are a result of its molecular structure which is that of a metal complex consisting of a ruthenium ion surrounded by three bipyridine ligands in an octahedral symmetry. ${ }^{17} \mathrm{~A} \mathrm{Ru}(\mathrm{bpy})_{3}^{2+}$ molecule in its ground state $|1\rangle$ can, by absorbing UV light, undergo a transition into several excited states (straight gray arrows in Fig. 2). ${ }^{17,18}$ Our calculations included all states ( $|5\rangle$ to $|8\rangle$ ) whose frequencies were up to $\Delta \omega=2000 \mathrm{THz}$ apart from the laser frequency $\left(\omega_{L}=5300 \mathrm{THz}\right.$, wavelength of $\lambda_{\mathrm{L}}=355 \mathrm{~nm}$ ). More distant states were not considered in the calculations as their influence to absorption and Raman scattering should be negligible (scaling with $1 / \Delta \omega^{224}$ ) and their inclusion would only increase the total computation time which for density matrix calculations generally scales with the square of the number of considered states. In Appendix A 4, it is discussed that a further reduction in the number of states in the model by combining states $|5\rangle-|8\rangle$ to a single effective state should not be performed, as it significantly changes the calculation results.

The population in one of the excited states $(|5\rangle-|8\rangle)$ relaxes with a rate of $20 \mathrm{ps}^{-1}$ into the lowest excited state but also with a much lower rate of $2 \mathrm{~ns}^{-1}$ back into the ground state. ${ }^{19}$ These transitions are indicated by wavy gray arrows in Fig. 2. The lowest excited state remains populated for a very long lifetime ( $890 \mathrm{~ns}$ if the molecule is in its common solvent acetonitrile ${ }^{20}$ ) before relaxing into the ground state and emitting fluorescence light around $600 \mathrm{~nm}$ wavelength. ${ }^{17}$

Further possible transitions (gray arrows in Fig. 2) exist from the lowest excited state to even higher electronic states $(|9\rangle-|11\rangle),{ }^{18}$ enabling Raman scattering from the lowest excited state. The lifetimes of these states are still unknown and in our level system assumed to be the same $2 \mathrm{~ns}$ as for the states $|5\rangle-|8\rangle$. This assumption should have no significant influence on the calculations, as the states receive only a small 
amount of population even at the highest studied pulse energy fluences.

As the lowest excited state $|3\rangle$ is a result of a metal-toligand charge transfer transition, ${ }^{17,21}$ the vibrational behavior of molecules in this state resembles the one of the individual ligands. This is the reason for the extensively studied spectral differences between the ground and the excited state Raman scattering of $\mathrm{Ru}(\mathrm{bpy})_{3}^{2+} \cdot 10,22,23$

Both the ground and the excited state Raman spectrum feature various vibrational resonances. However, the GSD as well as the Raman scattering into each of the individual vibrational states should both be independent from the number of modeled vibrational states as long as those states itself are only populated to a small extent (in total up to approximately $10 \%$ in the presented calculations). Therefore, it was assumed that including only two representative vibrational states as sources of the ground and excited state Raman scattering in our level system should not change the studied processes but provided the benefit of a reduced computation time. This claim was verified by investigations on the dependence of the Raman scattering on the number of modeled vibrational states reported in Appendix A 4.

All other investigations were performed with two vibrational states in the level system. One of those is state $|2\rangle$ at a frequency of $\omega_{2}=249 \mathrm{THz}$, corresponding to a wavenumber of $\tilde{v}_{2}=1320 \mathrm{~cm}^{-1}$, which can be populated by Raman scattering from the ground state $|1\rangle$ via virtual states nearby the electronic states $|5\rangle-|8\rangle$. The second state is state $|4\rangle$ at a frequency difference of $\omega_{4}-\omega_{3}=242 \mathrm{THz}$, corresponding to a wavenumber of $\tilde{v}_{4}=1284 \mathrm{~cm}^{-1}$, which can be populated by Raman scattering from the lowest excited state $|3\rangle$ via virtual states around the electronic states $|9\rangle-|11\rangle$.

Lifetimes of vibrational states were reported ranging from tens of picoseconds ${ }^{25}$ up to the nanosecond scale ${ }^{26}$ in the past. In our calculations, we assumed lifetimes of $1 \mathrm{~ns}$ for both vibrational states. This choice is consistent with earlier studies $^{12,15,16}$ and enabled to investigate the impact of population transfer to different numbers of vibrational states under the assumption that the population remained in those states on a time scale longer than the pulse duration (see Appendix A 4). It will be shown in Appendix A 5 that, for most calculations, the absolute value of the vibrational lifetime has only a minor influence on the calculated Raman scattering suppression.

Other important parameters for the density matrix calculations of $\mathrm{Ru}(\mathrm{bpy})_{3}^{2+}$ were the transition dipole moments $\mu_{p q}$ and the coherence lifetimes $\tau_{p q}^{\text {coh }}$ between the states. The latter were unfortunately unknown. In order to determine appropriate values, calculations were performed with various different coherence lifetimes and compared to the experimentally determined Raman scattering suppression in $\mathrm{Ru}(\mathrm{bpy})_{3}^{2+}$ as reported in Ref. 10 (see Sec. IV D). It was found that the assumption of a coherence lifetime of 2 ps between all states leads to an agreement with the experimental results so that this value was used in our model, although electronic coherence lifetimes of other photosynthetic molecules were determined to reach values of up to $200 \mathrm{fs}^{27}$ in the past.

Concerning the transition dipole moments, we experimentally determined $\mu_{51}$ to be $4.5 \mathrm{D}$ (with estimated uncertainty below $5 \%$ ) by measuring the absorption of $\mathrm{Ru}(\mathrm{bpy})_{3}^{2+}$ in the spectral region between $330 \mathrm{~nm}$ and $620 \mathrm{~nm}$ with a spectrophotometer and calculating the transition dipole moment from the acquired spectrum as described by Bertie $e t$ al. ${ }^{28}$ Our lab equipment did not allow for lower-wavelength absorption measurements nor for excited state absorption measurements. However, absorption spectra of all UV transitions originating from the ground state as well as from the lowest excited state are reported in Ref. 18. The reported complete absorption spectra were scaled such that the absorption peak of the transition from $|1\rangle-|5\rangle$ matched our measured absorption peak. From the scaled spectra, the values of the other transition dipole moments were calculated as listed in Table I (Appendix A 3).

For the calculations, it was assumed that the transition dipole moment between the vibrational state $|2\rangle$ and each electronic state $|k\rangle$ (with $k=5,6,7,8$ ) was equal to the transition dipole moment between the ground state $|1\rangle$ and this electronic state, i.e., $\mu_{k 2}=\mu_{k 1}$. The same assumption was made for the vibrational state $|4\rangle$, i.e., $\mu_{k 4}=\mu_{k 3}$ (with $k=9,10,11$ ). In reality, the two corresponding dipole moments may not be equal. However, the results of density matrix calculations in which the ratios of the transition dipole moments $\mu_{k 2} / \mu_{k 1}$ and $\mu_{k 4} / \mu_{k 3}$ were varied between 0.5 and 2 showed only a change of the pulse energy fluence required for a certain suppression of less than $40 \%$. These findings are in agreement with Ref. 16 which also reports only a small influence of these parameters on the population transfer dynamics in a numerical study on resolution enhancement in an SRS setup. Therefore, assuming the respective pairs of transition dipole moments to be equal should be reasonable for our model.

\section{CALCULATIONS OF RAMAN SCATTERING SUPPRESSION VIA GROUND STATE DEPLETION}

Density matrix calculations on a level system with a higher number of states, such as the one of Ru(bpy $)_{3}^{2+}$, cannot be performed with the simplified four-state density matrix differential equations ${ }^{13}$ used for earlier calculations. ${ }^{12-16}$ Therefore, we generalized the equations in order to allow the inclusion of any number of states (see Appendix A 1). Additionally, in order to enable a comparison between numerical and experimental results, the calculation of the spontaneous Raman scattering intensity from the density matrix calculations was established (see Appendix A 2).

The density matrix equations (A8) and (A9) (see Appendix A 1) of a $\mathrm{Ru}(\mathrm{bpy})_{3}^{2+}$ molecule assuming irradiation with a laser pulse at a wavelength of $355 \mathrm{~nm}$ were solved using a fourth-order Runge-Kutta algorithm. The calculations were performed with a time step width of $d t=0.1 \mathrm{fs}$ over a time frame that was four times the pulse duration used in the specific parameter set. In order to reduce the time to receive the calculation results, the variation of the pulse energy fluence was calculated in parallel on a multi-core computer.

Figure 3(a) shows exemplary results of a density matrix calculation using laser pulses with a duration of $\tau_{\text {pulse }}=30 \mathrm{ps}$ (intensity FWHM) and a pulse energy fluence of $4.9 \mathrm{~J} \mathrm{~cm}^{-2}$. The pulse energy fluence $\Phi=\left(\right.$ c n $\left.\epsilon_{0} \tau_{\mathrm{p}}\right) /(2 \zeta) \times \hat{E}^{2}$ was calculated from the peak amplitude $\hat{E}$ of the electric field, the pulse duration $\tau_{\mathrm{p}}$, the pulse form factor $\zeta=0.94$ (Gaussian pulse 

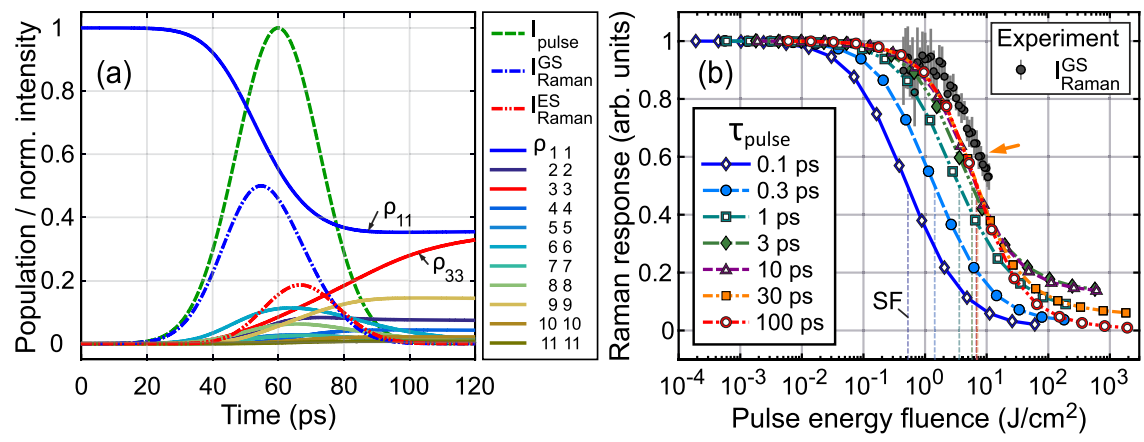

FIG. 3. Calculation of Raman scattering in $\operatorname{Ru}(\mathrm{bpy})_{3}^{2+}$. (a) Time development of the population $\rho_{p p}$ of all states $|p\rangle$ when irradiating the molecule with a pulse of 30 ps duration (intensity FWHM) at a wavelength of $355 \mathrm{~nm}$ and a pulse energy fluence of $4.9 \mathrm{~J} \mathrm{~cm}^{-2}$. Also displayed are the intensity of the incident laser pulse $I_{\text {pulse }}$ (dashed green curve, normalized to 1) and the ground and excited state Raman scattering intensities $I_{\text {Raman }}^{\mathrm{GS}}$ (dashed-dotted blue curve, normalized to 0.5) and $I_{\text {Raman }}^{\mathrm{ES}}$ (dashed-double-dotted red curve, scaled proportionally to the ground state Raman scattering). (b) Suppression of the ground state Raman response of $\mathrm{Ru}(\mathrm{bpy})_{3}^{2+}$ with increasing pulse energy fluence of a pulse at a wavelength of $355 \mathrm{~nm}$ for various different pulse durations $\tau_{\text {pulse. The curves were obtained }}$ point-by-point by calculating the time-integrated Raman scattering intensity of a calculation. An arrow points toward the specific marker resulting from the data shown in (a). In all calculations, the coherence lifetime between each pair of states was assumed to be $\tau_{p q}^{\text {coh }}=2$ ps. The suppression fluence (SF) is marked for each curve with a vertical line. Gray dots represent the experimental results from Ref. 10 for comparison.

shape), the speed of light $c$, the vacuum permittivity $\epsilon_{0}$, and the refractive index of the surrounding medium $n$. The latter was assumed to be $n=1.36$, as this is the refractive index of acetonitrile, a common solvent for $\mathrm{Ru}(\mathrm{bpy})_{3}^{2+}$, at a wavelength of $355 \mathrm{~nm}$. The temporal development of the population of all states is displayed, most importantly the ground state population $\rho_{11}$ (solid blue curve) and the lowest excited state population $\rho_{33}$ (solid red curve). It can be observed that the population is transferred from the ground state $|1\rangle$ to the electronic states $|5\rangle-|8\rangle$ by the leading edge of the incident laser pulse ( $I_{\text {pulse }}$, dashed green curve). The normalized Raman scattering intensity emitted from the ground state $\left(I_{\text {Raman }}^{\mathrm{GS}}\right.$, dashdotted blue curve) appears to be temporally shifted toward the leading edge of the pulse as the GSD, mainly present at the trailing edge, caused a suppression of the Raman scattering.

The relaxation of the population from the excited states $|5\rangle-|8\rangle$ (lifetime $20 \mathrm{ps}$ ) into the lowest excited state $|3\rangle$ occurs during the pulse duration ( $30 \mathrm{ps)}$ and continued afterwards. Therefore, Raman scattering from the lowest excited state ( $\mathrm{R}_{\mathrm{Raman}}^{\mathrm{ES}}$, dashed-double-dotted red curve) and the transfer of population from state $|3\rangle$ to the higher electronic states $\left(|9\rangle_{-}\right.$ $|11\rangle)$ were observable predominantly during the trailing edge of the pulse. Excited state Raman scattering is a characteristic property of $\mathrm{Ru}(\mathrm{bpy})_{3}^{2+17,22}$ but cannot be used for resolution-enhanced Raman microscopy and will, therefore, not be investigated in more detail in this publication.

The overall Raman scattering intensity from the ground state of the sample was obtained by integrating the timedependent signal over the considered time frame. By performing the calculation for different pulse energy fluences, it was found that the ground state Raman scattering signal increased linearly with the incident pulse energy fluence up to the point where GSD led to a saturation of this increase-an effect that can be applied for resolution-enhanced microscopy as described in Sec. II. This behavior can best be visualized by plotting the Raman response, i.e., the Raman scattering intensity relative to the incident pulse energy fluence, versus the pulse energy fluence as it is shown in Fig. 3(b) for pulses of different durations ranging from $0.1 \mathrm{ps}$ to $100 \mathrm{ps}$.
It can be seen that for all pulse durations, the Raman response is constant for a low pulse energy fluence and decreases at a higher pulse energy fluence before asymptotically approaching its minimal value. In the following, the minimal Raman response as well as the pulse energy required for a suppression of the Raman response by $50 \%$, henceforth called suppression fluence, will be used to quantify the efficiency of the Raman scattering suppression under varying conditions, e.g., for various different pulse durations. For a most efficient Raman scattering suppression in a potential resolutionenhanced microscopy application, both characteristic values should be as low as possible.

\section{A. Verification of the numerical model}

In order to verify that the density matrix calculations are able to reliably predict the behavior of molecules in an experimental realization of our proposed GSD Raman scattering spectroscopy, the numerical results have to be compared to the previously acquired experimental results of Ref. 10. The experimentally determined ground state Raman response is included for comparison in Fig. 3(b) as gray dots. The pulse energy fluence was calculated from the pulse energy used in the experiment focused to a diameter of $3.5 \mu \mathrm{m}$ (FWHM) with a Rayleigh length of $80 \mu \mathrm{m}$, considering a cuvette window transmission of $94 \%$ and a reduction of the laser intensity to approximately $45 \%$ after the Rayleigh length due to absorption in the sample $\left(10 \mathrm{mM}\right.$ concentration of $\left.\mathrm{Ru}(\mathrm{bpy})_{3}^{2+}\right)$.

In the experiment, a suppression of the ground state Raman response by $50 \%$ was achieved using laser pulses with a duration of $13 \mathrm{~ns}$ with a pulse energy of $1.5 \mu \mathrm{J}$ corresponding to a suppression fluence of $11 \mathrm{~J} \mathrm{~cm}^{-2}$. The calculations were restricted to pulse durations in the picosecond regime due to our limitations in computation time. As can be seen in Fig. 3(b), the Raman response curves shift toward higher pulse energy fluence with increasing pulse duration; however, this shift experienced a saturation for pulse durations above $3 \mathrm{ps}$. From this observation, it appeared to us as justified to compare our calculations carried out with 100 ps pulse duration with 
the experimental data, although the latter was obtained with 13 ns pulse duration.

It can be observed that the overall shape of the calculated ground state Raman response curve is in remarkable agreement with the experimental results, only slightly shifted toward a lower pulse energy fluence by a factor of approximately 1.6. The calculated suppression fluence was $8 \mathrm{~J} \mathrm{~cm}^{-2}$ in comparison to the experimental value of $11 \mathrm{~J} \mathrm{~cm}^{-2}$. This deviation can be attributed to a deviation of the real electronic coherence lifetimes from the assumed value of 2 ps (see Sec. IV D). Other possible parameters of influence are uncertainties in the measurement of the transition dipole moments, an imperfect estimation of the focal size in the experiment, and the fact that spatial variations of the light intensity due to the Gaussian intensity profile of the laser beam and decreasing laser intensity due to absorption are not considered in the density matrix calculations.

For the excited state Raman response, a comparison between the calculations and experiment cannot be expected to provide a quantitative agreement, as the shorter pulses used within the calculations experience a considerably lower population of the lowest excited state $|3\rangle$. This is due to this state receiving population on the time scale of the lifetime of the states $|5\rangle-|8\rangle$ (20 ps) such that the population transfer into this state is completed during the 13 ns laser pulses in the experiment, but not during the (up to) 100 ps laser pulses in the calculations [compare to Fig. 3(a)].

The complete set of molecular parameters that were used in the calculations to reproduce the experimental results from Ref. 10 as shown in Fig. 3(b) can be found in Appendix A 3.

\section{B. Influence of the pulse duration}

In order to choose a UV laser with an optimal pulse duration for a proposed application of GSD for resolutionenhanced microscopy, it is of high interest to gain insight into the dependence of the Raman scattering suppression on the pulse duration of the incident laser light. The dependence of the ground state Raman response on the pulse energy fluence was calculated for different pulse durations $\tau_{\text {pulse }}$ ranging from $0.1 \mathrm{ps}$ to $100 \mathrm{ps}$ covering three interesting time scales as shown in Fig. 3(b) and described in the following.

On the first time scale, the pulse duration (e.g., $\tau_{\text {pulse }}=100$ ps) exceeded the relaxation time of the higher excited states into the lowest excited state $(20 \mathrm{ps})$, while still being much shorter than the lifetime of the lowest excited state $(890 \mathrm{~ns})$. For such pulses, the minimal Raman response approached zero with increasing pulse duration as more population was transferred into the lowest excited state, achieving a complete GSD. The suppression fluence was $7.6 \mathrm{~J} \mathrm{~cm}^{-2}$ for a pulse duration of $100 \mathrm{ps}$. As the absolute amplitude of the Raman response [plotted normalized in Fig. 3(b)] is proportional to the pulse duration, it was higher for such longer pulses.

On the second time scale ( $\tau_{\text {pulse }}$ around a few picoseconds), the pulse duration was shorter than the relaxation time but longer than the coherence lifetime between pair of states (assumed to be $2 \mathrm{ps}$ ), resulting in a Raman scattering suppression with a comparable suppression fluence but a higher value of the minimal Raman response. This observation was a result of an equal distribution of population between the ground and several excited states allowing for a maximum suppression of the ground state Raman scattering signal down to a value that was proportional to the inverse of the number of involved states. The effect will be discussed in detail in Sec. IV D.

Finally, pulses shorter than the coherence lifetime between the excited states and the ground state $\left(\tau_{\text {pulse }}\right.$ of a few hundred femtoseconds) again accomplished a complete Raman scattering suppression with a minimal Raman response approaching zero. The suppression fluence was by approximately one magnitude lower in comparison to the one of longer pulses $\left(0.6 \mathrm{~J} \mathrm{~cm}^{-2}\right.$ for a pulse duration of $\left.100 \mathrm{fs}\right)$. However, this more efficient Raman scattering suppression comes with the disadvantage that it remains only on the time scale of the pulse duration (also shown in Sec. IV D). Additionally, using shorter pulses would provide a lower Raman scattering signal and, due to the conservation of the timebandwidth product, a reduced spectral resolution (approximately $4.4 \mathrm{THz}$ for $100 \mathrm{fs}$ pulses). Nevertheless, it was of interest to study the Raman suppression by short pulses as a method to gain insight into the general dynamics of GSD as well as to prepare the concept of GSD to be transferred to coherent Raman scattering methods which often employ short pulses in order to increase the nonlinear Raman scattering signal.

In general, the investigation on the influence of the pulse duration on the Raman response shows that a most efficient Raman scattering suppression (low suppression fluence and low minimal Raman response) can be achieved by avoiding the use of pulses with a duration shorter than the lifetime of the electronic states and longer than the coherence lifetime between ground and electronic states. Therefore, pulses shorter than the electronic coherence lifetime should be best suited for the suppression of the spontaneous Raman scattering. However, if longer pulses are required to generate the spontaneous Raman scattering, e.g., for reasons of the detection efficiency or spectral resolution, their duration should be longer than the lifetime, but in order to avoid a population relaxation back into the ground state on the time scale of the pulse duration, not longer than the lifetime of the lowest excited state (890 ns).

\section{Investigation of the possible resolution enhancement}

In order to investigate the possible resolution enhancement that could be achieved in an application of GSD in spontaneous Raman scattering microscopy, we calculated the image of a scattering center with the properties of $\mathrm{Ru}(\mathrm{bpy})_{3}^{2+}$. This process is described in detail in Ref. 10. Briefly, the Raman response of a sample irradiated with light of a certain pulse energy fluence was determined using the results of the density matrix calculations shown in Fig. 3(b). The scattering center (50 $\mathrm{nm}$ diameter) was numerically scanned (10 $\mathrm{nm}$ resolution) and the spatially integrated Raman scattering intensity calculated with three beams: a donut-shaped beam, a ten-fold lower intensity Gaussian beam, and a beam that was a combination of those two beams. By subtracting the images resulting from scanning with the donut-shaped and the combined beam from each other, a resolution-enhanced image was obtained as described in Sec. II. 
(a) Gaussian

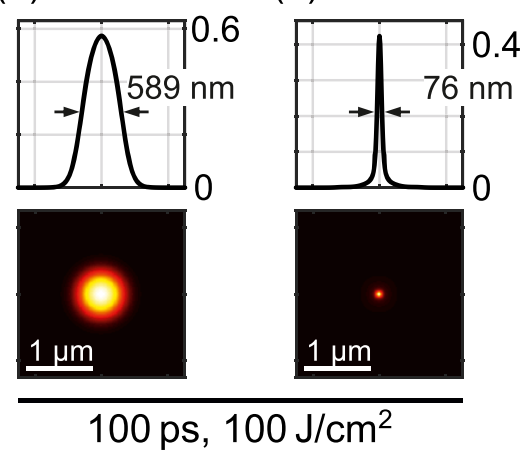

(c) Gaussian

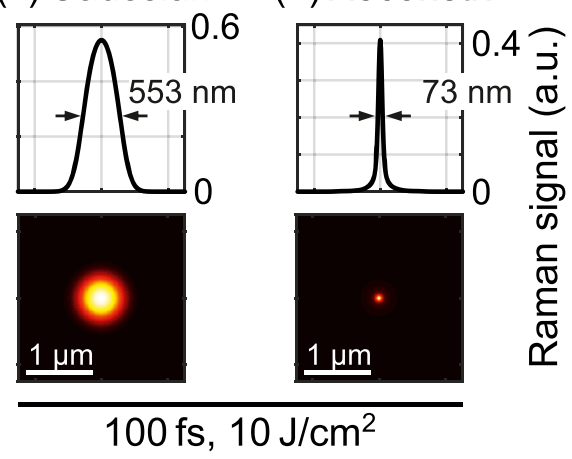

FIG. 4. Calculations of resolution enhancement via GSD assuming a scattering center emitting Raman scattering as it was calculated for Ru(bpy) ${ }_{3}^{2+}$ molecules irradiated with laser pulses with a duration of [(a) and (b)] $100 \mathrm{ps}$ and [(c) and (d)] $100 \mathrm{fs}$. All images are depicted as false-color 2D representation (bottom) and an additional 1D cut through their center (top). [(a) and (c)] Point spread functions (PSFs) obtained by scanning the sample with a diffraction-limited Gaussian beam. [(b) and (d)] Calculated resolution-enhanced image. For these calculations, the maximum local pulse energy fluence of the donut-shaped beams was set to $100 \mathrm{~J} \mathrm{~cm}^{-2}$ for (b) and $10 \mathrm{~J} \mathrm{~cm}^{-2}$ for (d), both resulting in a local suppression of the Raman response of approximately $94 \%$. The pulse energy fluence in the center of the Gaussian beam was $10 \mathrm{~J} \mathrm{~cm}^{-2}$ for (a) and $1 \mathrm{~J} \mathrm{~cm}^{-2}$ for (c). Note that the PSFs in (a) and (c) exhibit a slightly different width as the calculations were performed with different pulse energy fluences and based on different Raman response curves.

Figure 4 shows a comparison between the diffractionlimited images obtained by scanning the sample with a Gaussian beam in (a) and (c) as well as the resolution-enhanced difference image in (b) and (d). The image pairs [(a) and (b)] were calculated assuming pulsed laser irradiation with a pulse duration of $100 \mathrm{ps}$. The maximum local pulse energy fluence of the donut-shaped beam was set to $100 \mathrm{~J} \mathrm{~cm}^{-2}$. For the Gaussian beam, a ten-fold lower maximum local pulse energy fluence of $10 \mathrm{~J} \mathrm{~cm}^{-2}$ was used in order to avoid a broadening of the Gaussian form of the resulting point spread function (PSF) due to the nonlinear relation between the Raman scattering intensity and the incident pulse energy fluence. By comparing the two images, the theoretically possible resolution enhancement can be estimated to be approximately a factor of 7.5 (FWHM of $589 \mathrm{~nm}$ versus $76 \mathrm{~nm}$ ). Note, however, that such a high pulse energy fluence could not be used in an experimental realization of GSD as it is above the damage threshold of the molecules (approximately $15 \mathrm{~J} \mathrm{~cm}^{-2}$ ). ${ }^{10}$

The images shown in Figs. 4(c) and 4(d) were calculated based on the numerically obtained Raman response curve from Fig. 3(b) using $100 \mathrm{fs}$ pulses. When using femtosecond pulses, the pulse energy fluence required for the Raman scattering suppression was by approximately one order of magnitude lower in comparison to the one required when using picosecond pulses. Therefore, the two images also show a resolution enhancement by approximately a factor of 7.5 , although the maximum local pulse energy fluence of the donut-shaped and Gaussian beams in the calculations was set to only $10 \mathrm{~J} \mathrm{~cm}^{-2}$ and $0.6 \mathrm{~J} \mathrm{~cm}^{-2}$, respectively. Such pulse energy fluences are well below the damage threshold of $\mathrm{Ru}(\mathrm{bpy})_{3}^{2+}$ molecules, ${ }^{10}$ providing an incentive to further study the application of short femtosecond pulses for the GSD in future experiments also for other molecules, which would be more relevant for the life sciences.

The average laser power and intensity that we applied for GSD in $\mathrm{Ru}(\mathrm{bpy})_{3}^{2+}$ in Ref. $10\left(0.75 \mathrm{~mW}\right.$ and $\left.5.3 \mathrm{~kW} \mathrm{~cm}{ }^{-2}\right)$ are already two to three orders of magnitude lower than what was reported in the past for biological imaging (e.g., 80 $\mathrm{mW}$ with $60 \mathrm{~mW} \mathrm{~cm} \mathrm{~cm}^{-2}$ in the SRS beams in Ref. 29). On the other hand, due to our rather long (about $10 \mathrm{~ns}$ ) pulse duration, our pulse energy fluence was much higher (approximately $10 \mathrm{~J} \mathrm{~cm}^{-2}$ in Ref. 10 in comparison to $0.76 \mathrm{~J} \mathrm{~cm}^{-2}$ in Ref. 29). The comparison shows that the above-described suppression of Raman scattering might be possible with much shorter pulses, such as with one or more orders of magnitude lower pulse energy fluence. The option to work with much weaker radiation might then render resonant suppression of Raman scattering applicable for other molecules as well.

\section{Influence of the coherence lifetime}

As exact coherence lifetimes between the states of $\mathrm{Ru}(\mathrm{bpy})_{3}^{2+}$ are still unknown but were estimated-based on the comparison between numerical calculations and experimental results - to be $2 \mathrm{ps}$, a study on the influence of the variation of this value on the Raman scattering suppression was performed. For this purpose, calculations of the dependence of the Raman response on the pulse energy fluence were performed with varying coherence lifetimes between all states in the range from $20 \mathrm{ps}$, which is the lifetime of the electronic states of $\mathrm{Ru}(\mathrm{bpy})_{3}^{2+}$, down to $20 \mathrm{fs}$, which is five times shorter than the shortest pulse duration (100 fs) studied in Sec. IV B.

In order to gain insight into the influence of the coherence lifetime between the states on the suppression of the Raman response from pulses longer than each of the investigated coherence lifetimes, calculations were performed with pulses of 30 ps duration. A second set of calculations was performed with pulses of $100 \mathrm{fs}$ duration in order to investigate the influence of the coherence lifetime on the Raman response from pulses with a duration in the order of the coherence lifetime.

The coherence lifetimes between all states were varied equally, although in nature the coherence lifetimes would most likely differ between the individual pairs of states. This was a reasonable simplification in order to perform the calculations within a manageable parameter space. A brief discussion on 

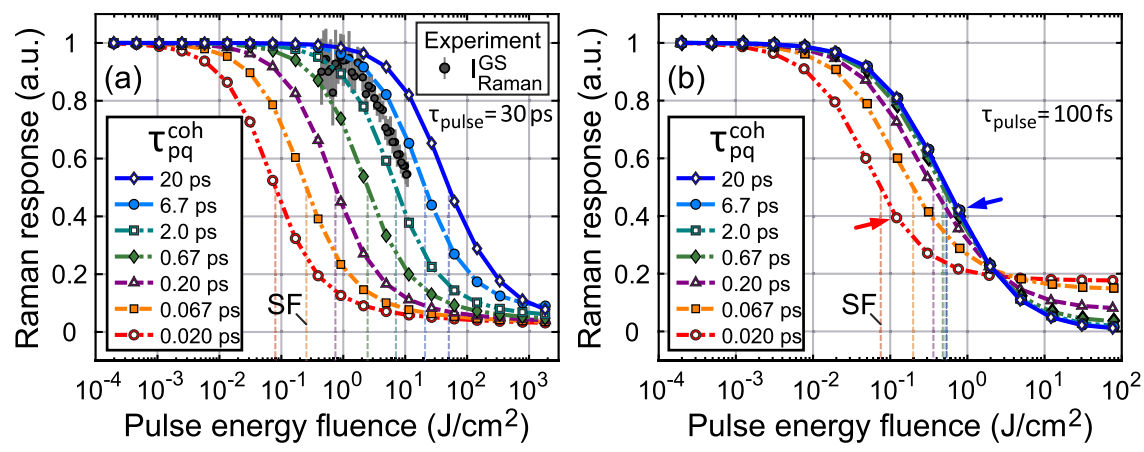

FIG. 5. Suppression of the ground state Raman response in Ru(bpy ${ }_{3}^{2+}$ with increasing pulse energy fluence for different coherence lifetimes $\tau_{p q}^{\text {coh }}$. Each symbol represents the time-integrated Raman scattering intensity obtained from a density matrix calculation performed with a laser pulse at a wavelength of 355 nm with a pulse duration of (a) $30 \mathrm{ps}$ and (b) $100 \mathrm{fs}$. The suppression fluence (SF) is marked for each curve with a vertical line. Gray dots represent the experimental results from Ref. 10 for comparison. The two arrows indicate the markers corresponding to the calculations shown in Fig. 6.

effects occurring when assuming different coherence lifetimes for the individual transitions can be found in Appendix A 6 .

Figure 5 shows the dependence of the Raman scattering suppression on the coherence lifetimes between the states calculated in (a) for 30 ps pulses and in (b) for $100 \mathrm{fs}$ pulses. The ground state Raman response integrated over the pulse duration is plotted versus the pulse energy fluence for each investigated coherence lifetime $\tau_{p q}^{\mathrm{coh}}$.

It can be seen in Fig. 5(a) that, when using picosecond pulses, a lower coherence lifetime resulted in a significant reduction (approximately two orders of magnitude) of the suppression fluence, i.e., $8 \times 10^{-2} \mathrm{~J} \mathrm{~cm}^{-2}$ instead of $5 \times 10^{1} \mathrm{~J} \mathrm{~cm}^{-2}$ for coherence lifetimes of $20 \mathrm{fs}$ and $20 \mathrm{ps}$, respectively. The minimal Raman response, however, remained unchanged by a variation of the coherence lifetimes. A possible physical explanation for the observed dependence of the suppression fluence could be that short electronic coherence lifetimes result in a spectral broadening of the molecular transitions, which leads to a more efficient GSD at the off-resonance laser wavelength of $355 \mathrm{~nm}$.

The gray dots in Fig. 5(a) mark the experimentally determined ground state Raman response from Ref. 10 as described in Sec. IV A. By comparing the experimental results to the numerical calculations, one can identify the coherence lifetime that is best suited to reproduce the experimental results. A value of 2 ps was chosen as the corresponding Raman suppression curve was close to the experimental data. The remaining difference in the suppression fluence could thenas described in Sec. IV A-be attributed to the experimental impact of absorption or intensity variations over the focus profile.

From the calculations shown in Fig. 5(b), it can be seen that also for femtosecond pulses, the suppression fluence was lower for shorter coherence lifetimes. However, the minimal Raman response also increased with decreasing coherence lifetimes. The reason for this dependence of the Raman response of femtosecond pulses is evident from the comparison of Figs. 6(a) and 6(b) which show two examples of the temporal development of the population of states (solid curves) for a molecule irradiated with a laser pulse (intensity $I_{\text {pulse }}$, dashed green curve) of 100 fs (intensity FWHM) duration assuming coherence lifetimes between the molecular states of $20 \mathrm{ps}$ and $20 \mathrm{fs}$, respectively. The pulse energy fluence used in the calculations was $0.8 \mathrm{~J} \mathrm{~cm}^{-2}$ and $0.1 \mathrm{~J} \mathrm{~cm}^{-2}$ for (a) and (b), respectively, resulting in a suppression of the Raman response to approximately 0.4 in both cases [see arrows in Fig. 5(b)].

For a coherence lifetime of 20 ps [see Fig. 6(a)], which is by a factor of 200 longer than the pulse duration, the population $\rho_{11}$ (blue curve) of the ground state $|1\rangle$ was transferred to the excited states $|5\rangle-|8\rangle$ during the first half of the pulse duration. At the center of the pulse, the ground state
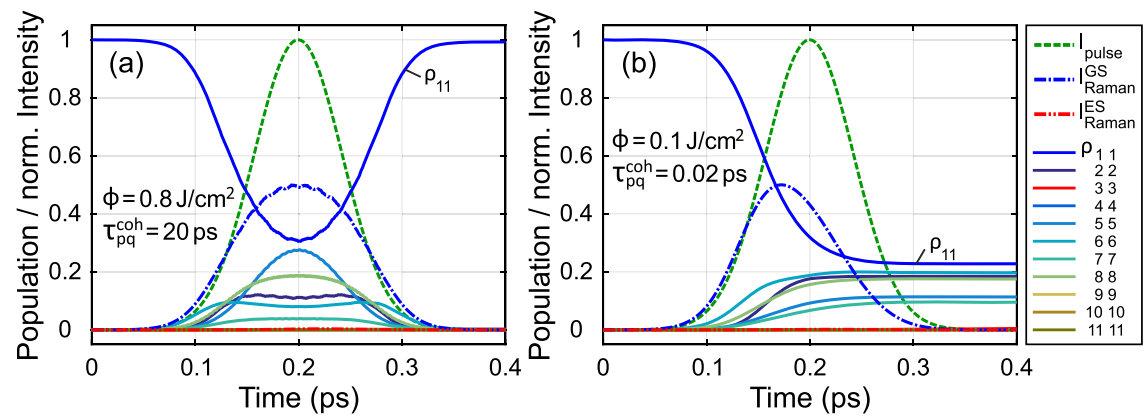

FIG. 6. Temporal development of the population of states of $\mathrm{Ru}(\mathrm{bpy})_{3}^{2+}$ irradiated with a laser pulse with a pulse duration of $100 \mathrm{fs}$ at a wavelength of $355 \mathrm{~nm}$. (a) Time development of the populations $\rho_{p p}$ of all states $|p\rangle$ assuming a coherence lifetime of $\tau_{p q}^{\text {coh }}=20 \mathrm{ps}$ and a pulse energy fluence of $0.8 \mathrm{~J} \mathrm{~cm}^{-2}$. Also displayed are the normalized intensity of the incident laser pulse $I_{\text {pulse }}$ (dashed green curve, normalized to unity) as well as the normalized ground and excited state Raman scattering intensities $I_{\text {pulse }}^{\mathrm{GS}}$ (dashed-dotted blue curve, normalized to 0.5 ) and $I_{\text {pulse }}^{\mathrm{ES}}$ (dashed-double-dotted red curve, scaled proportionally to the ground state Raman scattering). (b) The same as in (a) assuming a coherence lifetime of $\tau_{p q}^{\mathrm{coh}}=20 \mathrm{fs}$ and a pulse energy fluence of $0.1 \mathrm{~J} \mathrm{~cm}^{-2}$. 
population and with it the Raman scattering intensity exhibited an extremely fast modulation with low amplitude that was caused by Rabi cycling due to a buildup of coherence between the ground state and the excited states over the pulse duration. The population was transferred back to the ground state during the second half of the pulse duration which can be attributed to the effect of adiabatic following. ${ }^{30}$ As the Raman scattering intensity $I_{\text {Raman }}^{\mathrm{GS}}$ (dashed-dotted blue curve) is proportional to the population of the ground state [see Eq. (A11) in Appendix A 2], the maximum Raman scattering suppression was achieved in the center of the pulse, while the leading and trailing pulse edges experienced a lower suppression.

The above-described population transfer dynamics were also observed in the calculations discussed in Sec. IV B, using pulses shorter than the assumed 2 ps coherence lifetime resulting in the dependence of the Raman response on the pulse energy fluence that is depicted in Fig. 3(b) for those pulse durations.

Figure 6(b) shows that a coherence lifetime of $20 \mathrm{fs}$, which is by a factor of five shorter than the pulse duration, led to a longer-lasting transfer of the ground state population to the excited states $|5\rangle-|8\rangle$ as well as to the Raman state $|2\rangle$ due to Raman scattering. After the pulse, each of these molecular states was occupied to an almost equal amount and remained populated until the end of the considered time frame. More specifically, it can be seen that the GSD and, therewith, the Raman scattering suppression were proportional to the reciprocal number of the states which received a part of the total population from the ground state.

This type of population transfer dynamics was observed in the calculations discussed in Sec. IV B using pulses with a duration between the assumed coherence lifetime of $2 \mathrm{ps}$ and the population lifetime of 20 ps resulting in the dependence of the Raman response on the pulse energy fluence that was depicted in Fig. 3(b) for those pulse durations.

As can be seen in Fig. 5(b), the two investigated types of population dynamics are limit cases: When the coherence lifetime was in-between these examples, a part of the population was transferred back to the ground state and a part remained in the excited states causing the resulting Raman response curves to lie in-between the ones obtained for coherence lifetimes of $20 \mathrm{ps}$ and $20 \mathrm{fs}$. The pulse duration at which the transition between the two cases can be observed is defined by the coherence lifetime of the states.

The results of the above-discussed investigations have several consequences for potential applications: When studying molecules whose transitions posses lower coherence lifetimes with picosecond pulses, it should be possible to achieve a suppression of the Raman scattering with significantly lower pulse energy fluence (more than two orders of magnitude across the investigated parameters' space). When using femtosecond pulses, which are shorter than the lifetimes of the states, molecules with transitions whose coherence lifetimes are longer than the pulse duration would provide a lower minimal Raman response and, as a consequence, would allow for a higher resolution enhancement.

\section{E. Influence of the laser frequency}

In the past, investigations on the excited state Raman spectra of $\mathrm{Ru}(\mathrm{bpy})_{3}^{2+}$ were performed using laser pulses at a wavelength of $355 \mathrm{~nm}$ due to the convenient availability of this wavelength from frequency-tripled Neodymium:YAG lasers and their established application in resonance-enhanced Raman scattering spectroscopy. However, another wavelength, e.g., one that is closer to resonance with one of the transitions of $\mathrm{Ru}(\mathrm{bpy})_{3}^{2+}$, might lead to a more efficient GSD, motivating to study the influence of the laser wavelength on this process. Using our level system of $\mathrm{Ru}(\mathrm{bpy})_{3}^{2+}$ with multiple electronic states for the density matrix calculations enabled to detune the laser frequency toward the frequencies of those states and investigate the resulting Raman response.

For the following investigations, density matrix calculations were performed with different laser wavelengths (280 $\mathrm{nm}-470 \mathrm{~nm}$ ) covering the spectral region including the transitions to the states $|5\rangle-|8\rangle$. The Raman response curves were calculated with different pulse energy fluences $\left(1 \times 10^{-14} \mathrm{~J}\right.$ $\mathrm{cm}^{-2}-2 \times 10^{3} \mathrm{~J} \mathrm{~cm}^{-2}$ for the four on-resonance laser wavelengths and $7 \times 10^{-6} \mathrm{~J} \mathrm{~cm}^{-2}-2 \times 10^{3} \mathrm{~J} \mathrm{~cm}^{-2}$ for any other investigated wavelength). In all calculations, pulses with a duration of $30 \mathrm{ps}$ were used, as this pulse duration was closest to the experimental conditions, which still resulted in a reasonable computation time for this large parameter space. The suppression fluence was obtained from the resulting suppression curves and depicted in Fig. 7 as a function of the laser wavelength. The wavelengths corresponding to the four transitions between the ground state $|1\rangle$ and the excited states $|5\rangle-|8\rangle$ are marked with vertical gray bars.

Figure 7 reveals that the suppression fluence varied by more than six orders of magnitude over the considered wavelength range. The highest suppression fluence (approximately $15 \mathrm{~J} \mathrm{~cm}^{-2}$ ) was required in the region around $390 \mathrm{~nm}$ because this wavelength was most detuned from all resonances. The commonly used wavelength of $355 \mathrm{~nm}$ was close enough to the transition from $|1\rangle$ to $|6\rangle$ to achieve a slightly lower

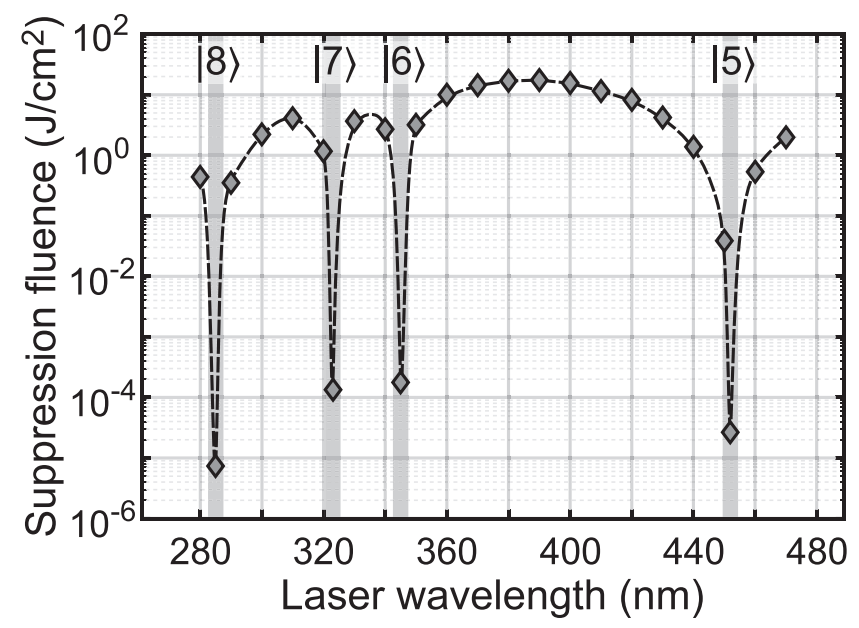

FIG. 7. Suppression fluence of the Raman scattering in dependence on the wavelength of the incident laser pulses. Symbols represent individual calculation results with lines being spline interpolations to guide the eye. Gray vertical bars represent the wavelengths corresponding to the transitions between ground state $|1\rangle$ and excited states $|5\rangle-|8\rangle$ as indicated. 
suppression fluence $\left(8 \mathrm{~J} \mathrm{~cm}^{-2}\right)$. The most efficient Raman scattering suppression with by more than four orders of magnitude lower suppression fluence $\left(7 \times 10^{-6} \mathrm{~J} \mathrm{~cm}^{-2}-2 \times 10^{-4} \mathrm{~J} \mathrm{~cm}^{-2}\right)$ was calculated for laser wavelengths that were in resonance with one of the four transitions from the ground state $|1\rangle$ to the states $|5\rangle-|8\rangle$ due to their high transition dipole moments $\left(\mu_{51}=4.5 \mathrm{D}, \mu_{61}=1.7 \mathrm{D}, \mu_{71}=2.0 \mathrm{D}\right.$, and $\left.\mu_{81}=8.2 \mathrm{D}\right)$ and due to the transition probability scaling with the inverse square of the detuning between laser and transition frequency. ${ }^{24}$ Additionally, a higher absolute intensity of the Raman scattering can be expected with a laser frequency close to resonance due to resonance-enhanced Raman scattering. ${ }^{31}$

In general, this investigation revealed that the pulse energy fluence required for the GSD can be significantly reduced by using a laser wavelength that is in resonance with one of the transitions in the molecule. This should also be true for molecules other than $\mathrm{Ru}(\mathrm{bpy})_{3}^{2+}$, which usually feature transitions in the UV, and should be considered in the design of potential future applications.

\section{FUTURE PERSPECTIVES}

\section{A. GSD in different molecules}

GSD Raman spectroscopy can most simply be applied to samples such as $\mathrm{Ru}(\mathrm{bpy})_{3}^{2+}$ that feature a long-lived excited state and which can be indirectly populated via strong transitions in order to excite an appreciable fraction of the ground state population. However, it is an important question, for instance, for microscopy purposes, in which other types of molecules' GSD can be achieved. Our investigations allow progressing toward an answer to this question by having analyzed two major aspects, in which typical Raman spectroscopy samples differ from samples such as $\mathrm{Ru}(\mathrm{bpy})_{3}^{2+}$.

First, most common molecules do not feature a similar long-lived excited state. In this point, our calculationsparticularly those in Secs. IV B and IV D-suggest that GSD would also be possible by directly populating excited states either by using a pulse duration below the electronic coherence lifetime to deplete the entire ground state on a short time scale or by populating multiple electronic states at once. The latter was shown to reduce the remaining ground state population to a fraction that is inversely proportional to the number of the populated states. ${ }^{15}$ Therefore, it should be sufficient for a sample to feature one or more states which can receive population from the ground state and have a lifetime which is longer than the duration of the pulses that are used to generate the Raman scattering.

The second important difference between $\mathrm{Ru}(\mathrm{bpy})_{3}^{2+}$ and other molecules concerns the minimum pulse energy fluence needed for GSD. Applying a long pulse duration and thus a high fluence can become a serious obstacle for GSD in other molecules, particularly in organic molecules with a lower damage threshold in comparison to $\mathrm{Ru}(\mathrm{bpy})_{3}^{2+}$. In our experiments, due to a very long pulse duration and with the laser somewhat detuned from resonance, we had to apply rather high fluence values of 5 to $10 \mathrm{~J} \mathrm{~cm}^{-2}$ although much smaller values seem sufficient for $\mathrm{Ru}(\mathrm{bpy})_{3}^{2+}$, particularly when using much shorter pulses. However, the pulse energy fluence to be applied in other molecules depends also on the dipole moments of the molecular transitions and will probably be higher than in $\mathrm{Ru}(\mathrm{bpy})_{3}^{2+}$. In order to maximize the Raman scattering suppression process for an application to different molecules, it is, therefore, crucial to find the minimum required pulse energy fluence. Our density matrix calculations suggest that it would be possible to achieve GSD with pulses that are even shorter than the electronic coherence lifetime (see Sec. IV B), especially when matching the laser wavelength to the resonances of the studied molecules (see Sec. IV E).

\section{B. GSD for CARS spectroscopy}

GSD should be applicable for a resolution enhancement not only in spontaneous but also in coherent Raman scattering microscopy, e.g., using CARS. We expect that the methods and results presented in this investigation are transferable considering that the spontaneous Raman scattering signal is proportional to the population of the ground state $\rho_{11}$, while that of CARS is proportional to the square of $\rho_{11} \cdot{ }^{15}$ Therefore, GSD is expected to lead to a stronger suppression of CARS signals than of signals generated by spontaneous Raman scattering.

In a potential resolution-enhanced CARS setup, Gaussianshaped pump and Stokes beams would have to be spatially superimposed, e.g., with donut-shaped control beams, generating the GSD around the focus to enhance the achievable spatial resolution. It appears to us as promising to realize such a scheme in the form of multiplex coherent anti-Stokes Raman scattering (M-CARS), ${ }^{32}$ in which a broadband (approximately $90 \mathrm{THz}$, corresponding to $3000 \mathrm{~cm}^{-1}$ ) Stokes pulse is used together with a narrowband (below $0.3 \mathrm{THz}$, corresponding to $10 \mathrm{~cm}^{-1}$ ) pump pulse and the entire spectrum of Raman scattered light is detected with a spectrometer. In M-CARS, it would be possible to temporally compress the broadband Stokes pulses to a duration in the femtosecond range so that it could be superimposed with a femtosecond UV control pulse. With such a scheme, one could exploit the numerically observed effect that GSD can be obtained with one order of magnitude lower pulse energy fluence when using femtosecond instead of picosecond control pulses. Thus, pulse energy fluences below $1 \mathrm{~J} \mathrm{~cm}^{-2}$ should be applicable, which are suited for biological imaging. ${ }^{29}$ In contrast to an application in spontaneous Raman scattering, the use of femtosecond pulses would not result in a loss of spectral resolution in such an MCARS scheme, as the spectral resolution would be determined by the bandwidth of the pump pulses.

\section{CONCLUSION}

In this publication, we presented density matrix calculations of the suppression of spontaneous Raman scattering by ground state depletion (GSD) in a level system based on the molecule tris(bipyridine)ruthenium(II) $\left(\mathrm{Ru}(\mathrm{bpy})_{3}^{2+}\right)$. The calculated Raman scattering suppression is in qualitative and reasonable quantitative agreement with earlier experimental results, ${ }^{10}$ verifying that Raman scattering processes from a molecule can be predicted by such calculations.

We assessed the required level of detail of the molecule model by comparing our calculations to the ones performed 
with more complex as well as oversimplified molecule models used in earlier work. ${ }^{13}$ It was found that simplifying the level system by neglecting certain electronic states leads to a substantial deviation of the resulting Raman response, whereas not including additional vibrational resonances in the model should be acceptable for most investigations.

Our calculations allowed us to identify pulse and molecule parameters which significantly influence the suppression of the Raman response, i.e., the pulse duration, the lifetimes of the molecular states, the coherence lifetimes between the states, and the laser wavelength.

The coherence lifetimes between the molecular states were found to significantly influence the necessary pulse energy fluence for the suppression of the Raman response so that reasonable assumptions of those values were required for the density matrix calculations.

Pulse durations that are either longer than the lifetime of the states ( $20 \mathrm{ps}$ ) or shorter than the coherence lifetime between the states (assumed 2 ps) resulted in a minimal Raman response close to zero and should be applicable for a resolution enhancement in spontaneous Raman scattering microscopy by up to a factor of 7.5 when using a maximum local pulse energy fluence of $100 \mathrm{~J} \mathrm{~cm}^{-2}$. In the case of the shorter femtosecond pulses, the Raman scattering suppression was obtained with a pulse energy fluence that is more than one order of magnitude lower (suppression fluence of $0.6 \mathrm{~J} \mathrm{~cm}^{-2}$ ) than the value observed in our recent corresponding experiment $\left(11 \mathrm{~J} \mathrm{~cm}^{-2}\right)$. With femtosecond pulses, a spatial resolution enhancement by up to a factor of 7.5 was calculated to be reached already with a maximum local pulse energy fluence of $10 \mathrm{~J} \mathrm{~cm}^{-2}$, about one-third below the damage threshold of the investigated molecule.

Finally, it was shown that using a laser wavelength that is in resonance with one of the electronic transitions of the sample should enable to suppress the Raman scattering with a by four to six orders of magnitude lower pulse energy fluence. Therefore, besides the other investigated parameters, also the laser wavelength should potentially be chosen with respect to the investigated sample, when designing potential future applications of GSD for resolutionenhanced Raman scattering microscopy. Additionally, our calculations provide a future perspective to numerically study the Raman scattering suppression via GSD in other molecules as well.

\section{ACKNOWLEDGMENTS}

This work was funded by the Cells-in-Motion Cluster of Excellence (EXC 1003-CiM) flexible fund Project No. FF2016-17. The authors thank Ernst-Ulrich Würthwein and Tim Hellwig for helpful discussions.

\section{APPENDIX: ADDITIONAL INFORMATION}

\section{Density matrix calculations using multi-state level systems}

Density matrix calculations can be used to predict the behavior of molecules under the influence of a light field. The general approach is well known and its application on a two-state system is in detail documented in Ref. 33. Reference 16 describes the application on a three-state system and Ref. 13 reports on the equations for a four-state system consisting of the ground state, a higher electronic state, and two vibrational states. Here, we briefly outline the derivation of general density matrix equations which can be applied to a molecular level system consisting of any number of states.

The molecule is described quantum mechanically with a density matrix $\tilde{\rho}$ consisting of elements $\tilde{\rho}_{p q}$ with $p, q=1,2, \ldots$, $N$, with $N$ being the number of molecular states included in the model. Each diagonal element $\tilde{\rho}_{p p}$ represents the population of a state $|p\rangle$, while each off-diagonal element $\tilde{\rho}_{p q}$ represents the coherence between two states, $|p\rangle$ and $|q\rangle$.

The time development of the density matrix is described by the Liouville equation ${ }^{33}$

$$
\frac{\mathrm{d}}{\mathrm{d} t} \tilde{\rho}=-\frac{i}{\hbar}[H, \tilde{\rho}]-L_{\text {relax }},
$$

with $\hbar$ being the reduced Planck constant, $H$ being the Hamiltonian of the system, and $L_{\text {relax }}$ being a matrix which accounts for classical non-radiative decay.

The elements of the Hamiltonian,

$$
\begin{aligned}
& H_{p p}=\hbar \omega_{p}, \\
& \underset{\substack{H_{p q} \\
p \neq q}}{H_{p q} E(t),}
\end{aligned}
$$

contain the energy $\hbar \omega_{p}$ of each state $|p\rangle$, the transition dipole moment $\mu_{p q}$ between two states, $|p\rangle$ and $|q\rangle$, and the electric field,

$$
E(t)=\frac{1}{2} \sum_{k}\left(E_{k}(t) \mathrm{e}^{-i \omega_{k} t}+\text { c.c. }\right),
$$

of the incident light which follows a classical description and consists of a sum of pulsed electric fields with carrier frequencies $\omega_{k}$ and pulse envelopes $E_{k}(t)$.

In order to simplify the numerical calculations of the resulting differential equations, a rotating-wave approximation $^{33}$ is applied to the density matrix elements, defining reduced density matrix elements

$$
\rho_{p q}=\tilde{\rho}_{p q} \mathrm{e}^{i \omega_{p q} t}
$$

with $\omega_{p q}=\omega_{p}-\omega_{q}$.

The relaxation matrix follows a classical description of population and coherence decay, respectively, and is given by

$$
\begin{aligned}
& L_{p p}=\sum_{k<p} R_{p k} \rho_{p p}-\sum_{k>p} R_{k p} \rho_{k k}, \\
& L_{p q}=\Gamma_{p q} \rho_{p q}
\end{aligned}
$$

with $R_{p q}$ being the population decay rate and $\Gamma_{p q}$ being the coherence decay rate between the states $|p\rangle$ and $|q\rangle$.

In order to reduce the numerical calculation time, the equations are restricted to only include the lower half $(p>q)$ of the density matrix and the diagonal elements $(p=q)$. The upper right half of the density matrix is subsequently calculated from the relation $\rho_{q p}=\rho_{p q}^{*}$.

Under these conditions, differential equations for the time development of the diagonal and off-diagonal density matrix 
elements can be derived from the Liouville equation (A1) to

$$
\begin{aligned}
\frac{\mathrm{d}}{\mathrm{d} t} \rho_{p p}= & -\frac{i}{2}\left[\sum_{k<p}\left(\chi_{p k} \rho_{p k}^{*}-\chi_{p k}^{*} \rho_{p k}\right)\right. \\
& \left.+\sum_{k>p}\left(\chi_{k p}^{*} \rho_{k p}-\chi_{k p} \rho_{k p}^{*}\right)\right]-\sum_{k<p} R_{p k} \rho_{p p}+\sum_{k>p} R_{k p} \rho_{k k},
\end{aligned}
$$$$
\begin{aligned}
\frac{\mathrm{d}}{\mathrm{d} t} \rho_{p>q}= & \frac{i}{2}\left[\chi_{p q}\left(\rho_{p p}-\rho_{q q}\right)+\sum_{k \neq p, q}\left(\chi_{q k}^{*} \rho_{p k}+\chi_{k q} \rho_{k p}^{*}\right.\right. \\
& \left.\left.-\chi_{k p}^{*} \rho_{k q}-\chi_{p k} \rho_{q k}^{*}\right)\right]-\Gamma_{p q} \rho_{p q},
\end{aligned}
$$

with the Rabi frequencies $\chi_{p q}$ defined as

$$
\chi_{p q}= \begin{cases}\mu_{p q} / \hbar \sum_{k} E_{k}(t) \mathrm{e}^{i\left(\omega_{p q}-\omega_{k}\right) t} & \text { if } p>q, \\ 0 & \text { if } p<q\end{cases}
$$

so that only terms with slowly varying electric fields in Eq. (A9) remain.

The density matrix equations (A8) and (A9) are solved by a fourth-order Runge-Kutta method ${ }^{34}$ in order to obtain the temporal development of the density matrix elements $\rho_{p p}$ and $\rho_{p q}$. The calculations are performed using different light field parameters, e.g., the electric field amplitude, pulse duration, and frequency, enabling to study the dependence of the population distribution on these parameters.

\section{Spontaneous Raman scattering in density matrix calculations}

Density matrix calculations of a molecule irradiated with a light field provide insight into the temporal development of the population of the molecular states. From the calculated results, one can also obtain information about the emitted field intensity from light matter interaction such as spontaneous or coherent Raman scattering.

The intensity of a CARS signal can be obtained from the density matrix calculation via the Maxwell equations. ${ }^{12-15}$ The signal intensity of SRS can be extracted from the population change between the ground state and a single vibrational state under the assumption that the SRS process is predominant over competing processes like spontaneous Raman scattering and non-radiative decay. ${ }^{16}$

The spontaneous Raman scattering intensity $I_{\text {Raman }}^{p q}(t)$ emitted at a time $t$ via transitions from a state $|q\rangle$ into a vibrational state $|p\rangle$ mediated by electronic states $|k\rangle$ can be calculated from the results of a density matrix calculation by ${ }^{24,35}$

$$
\begin{aligned}
I_{\text {Raman }}^{p q}(t)= & \frac{8 \pi \omega_{\mathrm{St}}^{4}}{9 \hbar^{2} c^{4}} I_{\mathrm{L}}(t) N_{0} \rho_{q q}(t) \\
& \times\left|\sum_{k} \frac{\mu_{k p} \mu_{k q}}{\omega_{k q}-\omega_{\mathrm{L}}-\mathrm{i} \Gamma_{k q} / 2}+\frac{\mu_{k p} \mu_{k q}}{\omega_{k p}+\omega_{\mathrm{L}}-\mathrm{i} \Gamma_{k p} / 2}\right|^{2},
\end{aligned}
$$

with $\omega_{\mathrm{St}}$ being the frequency of the emitted Stokes-shifted light, $\omega_{\mathrm{L}}$ being the laser frequency, $\omega_{p q}$ being the frequency difference, $\mu_{p q}$ being the transition dipole moment, and $\Gamma_{p q}$ being the coherence decay rate between the states $|p\rangle$ and $|q\rangle, I_{\mathrm{L}}(t)$ being the intensity of the pump laser pulse, $N_{0}$ being the number of irradiated molecules, $\rho_{q q}(t)$ being the population density of the state $|q\rangle$, and $c$ being the speed of light.

All but one parameter in Eq. (A11) are known from the density matrix calculation, as they are either input parameters (i.e., the frequencies, lifetimes, transition dipole moments, and the pump field intensity) or calculation results [i.e., the population density $\left.\rho_{q q}(t)\right]$. The exception is the total number of irradiated molecules $N_{0}$ which is an unknown value but constant in time in a static sample. In order to calculate the spontaneous Raman scattering intensity that would be emitted by an ensemble of molecules via Eq. (A11), we chose to set $N_{0}$ and all constants to one, resulting in a value given in arbitrary units. Knowledge about the absolute spontaneous Raman scattering intensity was unnecessary, as only its relative dependence on different pulse and molecule parameters was studied.

\section{Molecule parameters of $\mathrm{Ru}(\mathrm{bpy})_{3}^{2+}$}

Table I summarizes the parameters of the molecular model of $\mathrm{Ru}(\mathrm{bpy})_{3}^{2+}$ used for the density matrix calculations. A Raman scattering suppression comparable to the experimentally determined one from Ref. 10 was obtained with a calculation using these molecular parameters together with a laser wavelength of $355 \mathrm{~nm}$ and a pulse duration of $30 \mathrm{ps}$ or longer as discussed in Sec. IV A.

\section{Influence of the number of modeled states}

Figure 8(a) shows the suppression of the ground state Raman response obtained from density matrix calculations performed with only state $|6\rangle$ as an excited state (blue triangles) in comparison to the results from the calculations discussed in Sec. IV with all four excited states $|5\rangle-|8\rangle$ (orange squares). All calculations were performed with laser pulses at a wavelength of $355 \mathrm{~nm}$ with a pulse duration of $30 \mathrm{ps}$. It can be observed that the neglection of the other three electronic states results in a Raman response curve with a suppression fluence that is by approximately a factor of two higher than the one obtained from the calculations with all four states due to the reduction of the excitation probability of the molecule resulting from this neglection.

In order to compensate for neglecting states $|5\rangle,|7\rangle$, and $|8\rangle$, calculations were performed in which the transition dipole moments $\mu_{61}$ and $\mu_{62}$ were increased from their original value of $1.7 \mathrm{D}$ (blue triangles) to $2.7 \mathrm{D}$ (purple circles), to $3.7 \mathrm{D}$ (cyan diamonds), and finally to $5.7 \mathrm{D}$ (green triangles). As can be seen, the use of transition dipole moments of slightly less than $2.7 \mathrm{D}$ in the calculations would lead to the suppression fluence matching closest to the one obtained from the calculations based on the complete model. However, due to the lack of additional electronic states accepting population from the ground state on the time scale of the pulse duration, the minimum Raman response was by approximately a factor of 2.5 higher.

From this investigation, it can be concluded that in general density matrix calculations with a reduced number of 
TABLE I. Considered states $|p\rangle$ of $\mathrm{Ru}(\mathrm{bpy})_{3}^{2+}$ with angular frequencies $\omega_{p}$, wavelengths $\lambda_{p q}$ (for electronic states), relative wavenumbers $\tilde{v}_{p q}$ (for vibrational states), transition dipole moments $\mu_{p q}$, and lifetimes $\tau_{p q}$ for transitions originating from or ending in state $|q\rangle$. Footnotes indicate the sources of the respective values.

\begin{tabular}{|c|c|c|c|c|}
\hline$|p\rangle$ & $\omega_{p}(\mathrm{THz})$ & $\lambda_{p q}$ or $\tilde{v}_{p q}$ & $\mu_{p q}$ & $\tau_{p q}$ \\
\hline$|11\rangle$ & $\omega_{11}=10543$ & $\lambda_{113}=251 \mathrm{~nm}^{\mathrm{a}}$ & $\mu_{113}=7.3 \mathrm{D}^{\mathrm{b}}$ & $\tau_{113}=2 \mathrm{~ns}^{\mathrm{c}}$ \\
\hline$|10\rangle$ & $\omega_{11}=9056$ & $\lambda_{103}=313 \mathrm{~nm}^{\mathrm{a}}$ & $\mu_{103}=4.0 \mathrm{D}^{\mathrm{b}}$ & $\tau_{103}=2 \mathrm{~ns}^{\mathrm{c}}$ \\
\hline$|9\rangle$ & $\omega_{9}=8157$ & $\lambda_{93}=368 \mathrm{~nm}^{\mathrm{a}}$ & $\mu_{93}=5.4 \mathrm{D}^{\mathrm{b}}$ & $\tau_{93}=2 \mathrm{~ns}^{\mathrm{c}}$ \\
\hline$|8\rangle$ & $\omega_{8}=6609$ & $\lambda_{81}=285 \mathrm{~nm}^{\mathrm{a}}$ & $\mu_{81}=8.2 \mathrm{D}^{\mathrm{b}}$ & $\tau_{81}=2 \mathrm{~ns}^{\mathrm{d}}, \tau_{83}=20 \mathrm{ps}^{\mathrm{d}}$ \\
\hline$|7\rangle$ & $\omega_{7}=5832$ & $\lambda_{71}=323 \mathrm{~nm}^{\mathrm{a}}$ & $\mu_{71}=2.0 \mathrm{D}^{\mathrm{b}}$ & $\tau_{71}=2 \mathrm{~ns}^{\mathrm{d}}, \tau_{73}=20 \mathrm{ps}^{\mathrm{d}}$ \\
\hline$|6\rangle$ & $\omega_{6}=5460$ & $\lambda_{61}=345 \mathrm{~nm}^{\mathrm{a}}$ & $\mu_{61}=1.7 \mathrm{D}^{\mathrm{b}}$ & $\tau_{61}=2 \mathrm{~ns}^{\mathrm{d}}, \tau_{63}=20 \mathrm{ps}^{\mathrm{d}}$ \\
\hline$|5\rangle$ & $\omega_{5}=4167$ & $\lambda_{51}=452 \mathrm{~nm}^{\mathrm{a}}$ & $\mu_{51}=4.5 \mathrm{D}^{\mathrm{e}}$ & $\tau_{51}=2 \mathrm{~ns}^{\mathrm{d}}, \tau_{53}=20 \mathrm{ps}^{\mathrm{d}}$ \\
\hline$|4\rangle$ & $\omega_{4}=3280$ & $\tilde{v}_{43}=1284 \mathrm{~cm}^{-1 \mathrm{f}}$ & $\mu_{k 4}=\mu_{k 3}$ & $\tau_{43}=1 \mathrm{~ns}^{\mathrm{g}}$ \\
\hline$|3\rangle$ & $\omega_{3}=3038$ & $\lambda_{31}=619 \mathrm{~nm}^{\mathrm{a}}$ & $\mu_{k 3}=\mu_{k 1}$ & $\tau_{31}=890 \mathrm{~ns}^{\mathrm{h}}$ \\
\hline$|2\rangle$ & $\omega_{2}=249$ & $\tilde{v}_{21}=1320 \mathrm{~cm}^{-1 \mathrm{f}}$ & $\mu_{k 2}=\mu_{k 1}$ & $\tau_{21}=1 \mathrm{~ns}^{\mathrm{g}}$ \\
\hline$|1\rangle$ & $\omega_{1}=0$ & Ground state & $\mu_{k 1}$ see above & $\mathrm{n} / \mathrm{a}$ \\
\hline
\end{tabular}

${ }^{\mathrm{a}}$ References 17 and 18 .

${ }^{\mathrm{b}}$ Data from Ref. 18 scaled on own measurement.

${ }^{\mathrm{c}}$ Assumed to be equal to $\tau_{5}$ to $\tau_{8} 1$.

${ }^{\mathrm{d}}$ Reference 19.

${ }^{\mathrm{e}}$ Calculated from measurement as described in Ref. 28.

${ }^{\mathrm{f}}$ Reference 10 and also Refs. 22 and 23.

$\mathrm{g}_{\text {Assumed based on Refs. 12, 15, and } 16 .}$

${ }^{\mathrm{h}}$ Reference 20 .

electronic states may not provide sufficiently accurate results even if the dipole moments of the remaining transitions are accordingly increased. Additionally, knowledge about the optimal values of the transition dipole moments that should be used in order to compensate for the simplification of the level system can only be obtained by performing calculations as shown in Fig. 8(a). Finally, by not including all electronic transitions in the model, its applications would be limited, e.g., it would not be possible to scan the laser frequency in respect to those transitions and thereby study the frequency dependence of the Raman scattering suppression as discussed in Sec. IV E.

Figure 8(b) shows a comparison of the ground state Raman response obtained from density matrix calculations performed with different numbers of vibrational states. The comparison is shown for two different pulse durations of $30 \mathrm{ps}$ (orange squares and red triangles) and $100 \mathrm{fs}$ (blue diamonds and bluegreen squares). Two of the depicted curves (orange squares and blue diamonds) are results of the calculations discussed in Sec. IV based on level systems with one vibrational resonance each of the ground and the excited state. The other two sets of three curves (red triangles and blue-green squares) are results of density matrix calculations using three ground and three excited state vibrational resonances.

The calculations were performed by adding additional states $|2 b\rangle,|2 c\rangle,|4 b\rangle$, and $|4 c\rangle$ to the level system which share the properties of states $|2\rangle\left(\tilde{v}_{2}=1320 \mathrm{~cm}^{-1}\right)$ and $|4\rangle$ $\left(\tilde{v}_{4}=1284 \mathrm{~cm}^{-1}\right)$, except for their frequencies which correspond to different resonances visible in the ground and excited state Raman spectrum of $\mathrm{Ru}(\mathrm{bpy})_{3}^{2+}$ at wavenumbers of $\tilde{v}_{2 b}=1493 \mathrm{~cm}^{-1}, \tilde{v}_{2 c}=1608 \mathrm{~cm}^{-1}, \tilde{v}_{4 b}=1427 \mathrm{~cm}^{-1}$, and $\tilde{v}_{4 c}=1550 \mathrm{~cm}^{-1}$.
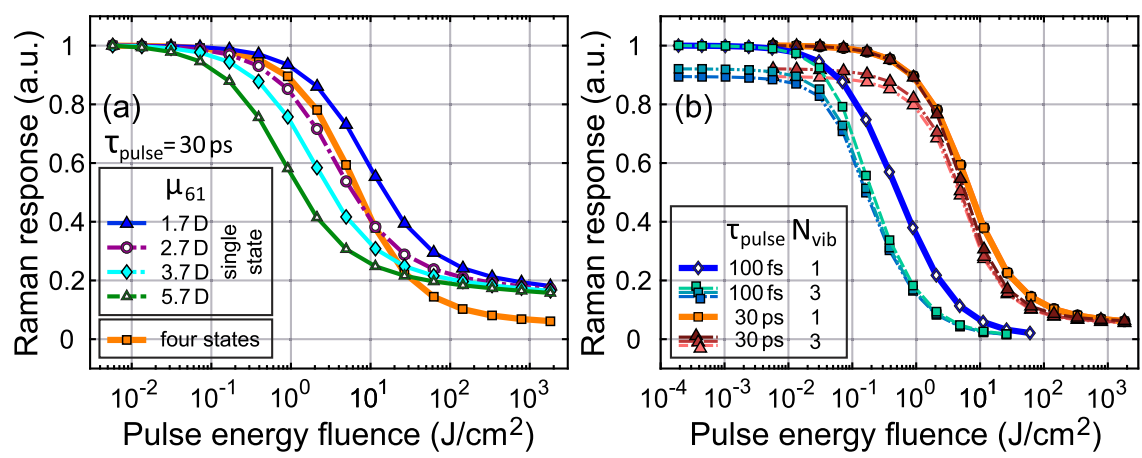

FIG. 8. Calculations of the suppression of the Raman response in Ru(bpy $)_{3}^{2+}$ with increasing pulse energy fluence based on level systems with different configurations of states. Orange squares and blue diamonds represent the results from Sec. IV obtained with 30 ps and 100 fs pulses, respectively, including the four electronic states $|5\rangle-|8\rangle$ and two single vibrational states $|2\rangle$ and $|4\rangle$ in the calculations. (a) Comparison to calculations using only a single electronic state |6) with varying transition dipole moments $\mu_{61}$ with $\mu_{62}=\mu_{61}$ (blue triangles, purple circles, cyan diamonds, green triangles). (b) Comparison to calculations employing a number of $N_{\text {vib }}=3$ vibrational states for both the ground and excited states (blue-green squares, red triangles). All calculations were performed with laser pulses at a wavelength of $355 \mathrm{~nm}$. 
In Fig. 8(b), the Raman response curves obtained from the calculations with three ground state vibrational resonances were normalized on the Raman response resulting from the transition to state $|2\rangle$. It can be observed that the maximum Raman response resulting from the transition to the states $|2 b\rangle$ and $|2 c\rangle$ is by around $10 \%$ lower due to the slightly higher difference between the frequencies of those states to the one of the ground state. A suppression of the Raman response with increasing pulse energy fluence is observed regardless whether the calculations used the simpler or the more complex level system. However, the suppression fluence is found to be slightly lower when using three vibrational states, more precisely by an approximate factor of 1.25 and 2.5 for $30 \mathrm{ps}$ and $100 \mathrm{fs}$ pulses, respectively.

The reason for this discrepancy is that additional vibrational states can only be neglected in the density matrix model, if the population transferred to the states is negligible. This would always be the case if the lifetimes of the vibrational states were much shorter than the pulse duration, causing an almost instantaneous relaxation of the population into the ground state. However, as a vibrational lifetime of $1 \mathrm{~ns}$ was assumed in the calculations, a single vibrational state was found to receive up to $11 \%$ of the population in the temporal center of the pulse [see Fig. 3(a)]. When including three states, each individual state received up to $8 \%$ of the total population. Therefore, a slightly less pulse energy fluence was required for the GSD. This effect is more influential when using femtosecond than picosecond pulses, as their time frame of Raman scattering emission has a larger overlap with the population of the vibrational states [compare Figs. 3(a) and 6(a)].

Knowing the positive influence of additional vibrational states on the suppression of the Raman scattering, which is low especially for picosecond pulses, our model of $\mathrm{Ru}(\mathrm{bpy})_{3}^{2+}$ was restricted to only a single vibrational resonance for the ground and the excited state in order to reduce the computation time.

\section{Influence of the vibrational lifetime}

As discussed in Sec. III, the exact lifetimes of the vibrational states $|2\rangle$ and $|4\rangle$ of $\mathrm{Ru}(\mathrm{bpy})_{3}^{2+}$ are unknown but were chosen to be $1 \mathrm{~ns}$ throughout all previous calculations in this

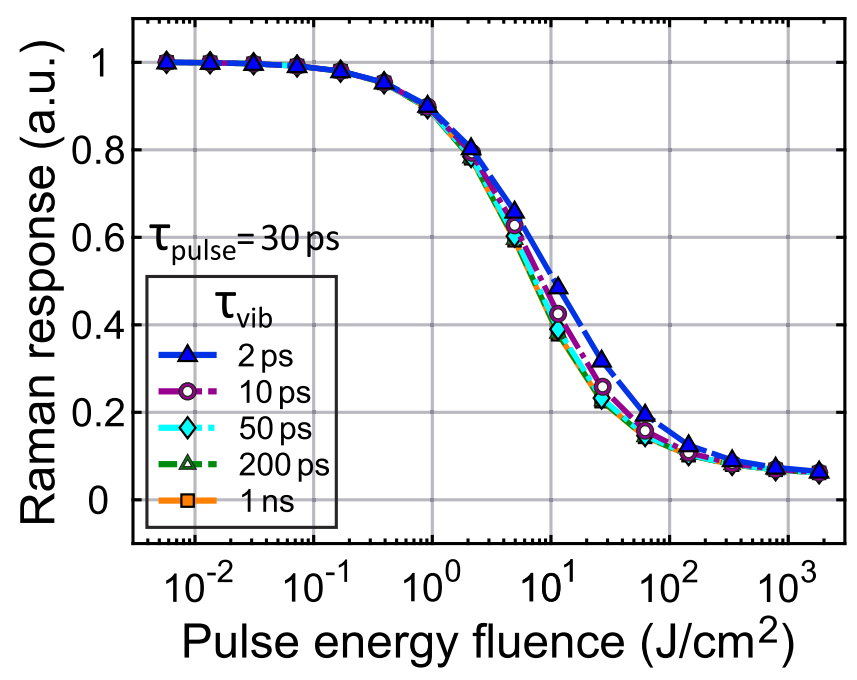

FIG. 9. Suppression of the ground state Raman response in $\mathrm{Ru}(\mathrm{bpy})_{3}^{2+}$ with increasing pulse energy fluence for varying vibrational lifetimes $\tau_{v i b}=1 / R_{21}$ $=1 / R_{43}$. Each symbol represents the time-integrated Raman scattering intensity obtained from a density matrix calculation performed with a laser pulse at a wavelength of $355 \mathrm{~nm}$ with a pulse duration of $30 \mathrm{ps}$.

study. In the following, density matrix calculations with different vibrational lifetimes will be shown in order to investigate the impact of the vibrational lifetime on the calculated Raman scattering suppression.

Figure 9 shows the ground state Raman response plotted versus the pulse energy fluence for a pulse duration of $30 \mathrm{ps}$ and different lifetimes of the vibrational states $\tau_{v i b}=1 / R_{21}$ $=1 / R_{43}$ between $2 \mathrm{ps}$ and $1 \mathrm{~ns}$, covering the range of reported vibrational lifetimes. ${ }^{25,26}$ Orange squares represent calculations with vibrational lifetimes of $1 \mathrm{~ns}$, identical to the results shown in Fig. 3(b). Other markers (green triangles, cyan diamonds, purple circles, and blue triangles) represent calculations with lower vibrational lifetimes as denoted. It can be observed that the Raman response is suppressed at approximately the same pulse energy fluence following a similar curve shape for all five calculations with different vibrational lifetimes. Only for vibrational lifetimes below the pulse duration (e.g., 2 ps, blue triangles), a minor increase of the suppression fluence can be observed. This is due to the fact that population transferred to the vibrational state $|2\rangle$ relaxes back to
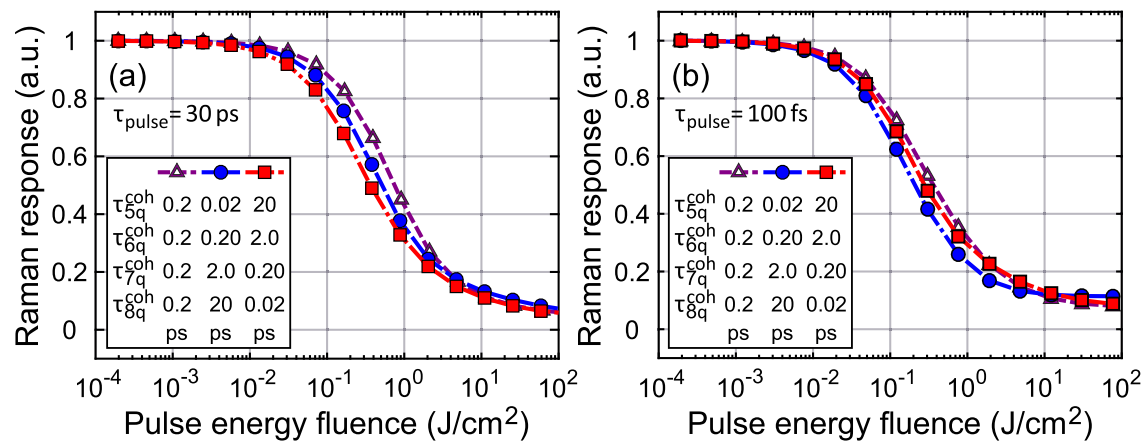

FIG. 10. Suppression of the ground state Raman response in Ru(bpy $)_{3}^{2+}$ with increasing pulse energy fluence for varying coherence lifetimes $\tau_{p q}^{\text {coh }}$ of the molecular transitions. Each symbol represents the time-integrated Raman scattering intensity obtained from a density matrix calculation performed with a laser pulse at a wavelength of $355 \mathrm{~nm}$ with a pulse duration of (a) $30 \mathrm{ps}$ or (b) $100 \mathrm{fs}$. Purple triangles represent the results obtained with coherence lifetimes of 0.2 ps for all transitions. Blue dots and red squares result from calculations in which different coherence lifetimes were assumed between the states $|p\rangle=|5\rangle,|6\rangle,|7\rangle,|8\rangle$ and $|q\rangle$ with $(q<p)$ as indicated in the legend. 
the ground state during the pulse duration such that there is only a small contribution of the vibrational state to the overall GSD. However, this effect is small so that it can be concluded that the Raman scattering suppression is only insignificantly influenced by the exact choice of the assumed vibrational lifetime.

\section{Influence of transitions with different coherence lifetimes}

In the investigations discussed in Sec. IV D, the coherence lifetimes between the individual states were assumed to be equal and were varied uniformly. In order to give insight into the dependence of the Raman suppression on a non-uniform variation of the coherence lifetimes, density matrix calculations were performed in which different coherence lifetimes (between $20 \mathrm{fs}$ and $20 \mathrm{ps}$ ) were assumed for the transitions to the individual states $|5\rangle,|6\rangle,|7\rangle$, and $|8\rangle$.

Figure 10 shows the ground state Raman response obtained from these calculations performed with two different pulse durations [30 ps in (a) and $100 \mathrm{fs}$ in (b)]. Blue dots represent calculations in which the coherence lifetimes of the transitions from states $|p\rangle=|5\rangle,|6\rangle,|7\rangle,|8\rangle$ to states $|q\rangle$ with $(q<p)$ were assumed to be $\tau_{5 q}^{\text {coh }}=20 \mathrm{fs}, \tau_{6 q}^{\text {coh }}=200 \mathrm{fs}$, $\tau_{7 q}^{\mathrm{coh}}=2 \mathrm{ps}$, and $\tau_{8 q}^{\mathrm{coh}}=20 \mathrm{ps}$. In the calculations represented by red dots, the same coherence lifetimes were assumed in reversed order (see the legend of Fig. 10). It can be observed that the two Raman response curves closely resemble each other, although different distributions of coherence lifetimes were used within the calculations. These results imply that the suppression of the Raman response is apparently influenced stronger by the absolute values of the coherence lifetimes of the molecular transitions than by the distribution of those values among the individual transitions.

In order to allow for a comparison, purple triangles mark the results of the calculations discussed in Sec. IV D, in which the coherence lifetimes were assumed to be $\tau_{p q}^{\text {coh }}=0.2$ ps for all transitions. This curve shows a close resemblance to the other two obtained from the calculations with coherence lifetimes varying between $20 \mathrm{fs}$ and $20 \mathrm{ps}$. It can, therefore, be concluded that in a level system consisting of transitions with varying coherence lifetimes, low coherence lifetimes seem to dominate over the high ones so that the resulting Raman response curves match to curves which were calculated with only low coherence lifetimes. This effect is consistent with the observation that lower coherence lifetimes provided GSD already at a lower pulse energy fluence as discussed in Sec. IV D.
${ }^{1}$ S. W. Hell and J. Wichmann, Opt. Lett. 19, 780 (1994).

${ }^{2}$ M. J. Rust, M. Bates, and X. Zhuang, Nat. Methods 3, 793 (2006).

${ }^{3}$ E. Betzig, G. H. Patterson, R. Sougrat, O. W. Lindwasser, S. Olenych, J. S. Bonifacino, M. W. Davidson, J. Lippincott-Schwartz, and H. F. Hess, Science 313, 1642 (2006).

${ }^{4}$ F. Balzarotti, Y. Eilers, K. C. Gwosch, A. H. Gynnå, V. Westphal, F. D. Stefani, J. Elf, and S. W. Hell, Science 355, 606 (2017).

${ }^{5}$ H. Kim, G. W. Bryant, and S. J. Stranick, Opt. Express 20, 6042 (2012).

${ }^{6}$ A. Gasecka, A. Daradich, H. Dehez, M. Piché, and D. Côté, Opt. Lett. 38, 4510 (2013).

${ }^{7}$ W. R. Silva, C. T. Graefe, and R. R. Frontiera, ACS Photonics 3, 79 (2016).

${ }^{8}$ D. Kim, D. S. Choi, J. Kwon, S.-H. Shim, H. Rhee, and M. Cho, J. Phys. Chem. Lett. 8, 6118 (2017).

${ }^{9}$ M. Cho, J. Chem. Phys. 148, 014201 (2018).

${ }^{10}$ S. Rieger, M. Fischedick, K.-J. Boller, and C. Fallnich, Opt. Express 24, 20745 (2016).

${ }^{11}$ K.-J. Boller, W. Beeker, C. Cleff, K. Kruse, C. Lee, P. Groß, H. Offerhaus, C. Fallnich, and J. Herek, in Super-Resolution Microscopy Techniques in the Neurosciences, Volume 86 of Neuromethods, edited by E. F. Fornasiero and S. O. Rizzoli (Humana Press, 2014), Chap. 12, p. 291.

${ }^{12}$ W. P. Beeker, C. J. Lee, K.-J. Boller, P. Groß, C. Cleff, C. Fallnich, H. Offerhaus, and J. Herek, Phys. Rev. A 81, 012507 (2010).

${ }^{13}$ W. P. Beeker, P. Groß, C. J. Lee, C. Cleff, H. Offerhaus, C. Fallnich, J. Herek, and K.-J. Boller, Opt. Express 17, 22632 (2009).

${ }^{14}$ C. Cleff, P. Groß, C. Fallnich, H. L. Offerhaus, J. Herek, K. Kruse, W. P. Beeker, C. J. Lee, and K.-J. Boller, Phys. Rev. A 87, 033830 (2013).

${ }^{15}$ C. Cleff, P. Groß, C. Fallnich, H. L. Offerhaus, J. Herek, K. Kruse, W. P. Beeker, C. J. Lee, and K.-J. Boller, Phys. Rev. A 86, 023825 (2012).

${ }^{16}$ L. Gong and H. Wang, Phys. Rev. A 90, 013818 (2014).

${ }^{17}$ K. Kalyanasundaram, Coord. Chem. Rev. 46, 159 (1982).

${ }^{18}$ U. Lachish, P. P. Infelta, and M. Grätzel, Chem. Phys. Lett. 62, 317 (1979).

${ }^{19}$ F. E. Lytle and D. M. Hercules, J. Am. Chem. Soc. 91, 253 (1969).

${ }^{20}$ K. Nakamaru, Bull. Chem. Soc. Jpn. 55, 1639 (1982).

${ }^{21}$ T. J. Meyer, Acc. Chem. Res. 22, 163 (1989).

${ }^{22}$ R. F. Dallinger and W. H. Woodruff, J. Am. Chem. Soc. 101, 4391 (1979).

${ }^{23}$ P. A. Mabrouk and M. S. Wrighton, Inorg. Chem. 25, 526 (1986).

${ }^{24}$ W. Demtröder, Laser Spectroscopy (Springer, Berlin, Heidelberg, 2003).

${ }^{25}$ A. Laubereau, D. von der Linde, and W. Kaiser, Phys. Rev. Lett. 28, 1162 (1972).

${ }^{26}$ R. S. Urdahl, D. J. Myers, K. D. Rector, P. H. Davis, B. J. Cherayil, and M. D. Fayer, J. Chem. Phys. 107, 3747 (1997).

${ }^{27}$ N. Christensson, H. F. Kauffmann, T. Pullerits, and T. Mančal, J. Phys. Chem. B 116, 7449 (2012).

${ }^{28}$ J. E. Bertie, S. L. Zhang, and C. D. Keefe, Vib. Spectrosc. 8, 215 (1995).

${ }^{29}$ C. W. Freudiger, W. Min, B. G. Saar, S. Lu, G. R. Holtom, C. He, J. C. Tsai, J. X. Kang, and X. S. Xie, Science 322, 1857 (2008).

${ }^{30}$ G. L. Peterson and C. D. Cantrell, Phys. Rev. A 31, 807 (1985).

${ }^{31}$ E. V. Efremov, F. Ariese, and C. Gooijer, Anal. Chim. Acta 606, 119 (2008).

${ }^{32}$ G. W. H. Wurpel, J. M. Schins, and M. Müller, Opt. Lett. 27, 1093 (2002).

${ }^{33}$ P. W. Milonni and J. H. Eberly, Laser Physics (John Wiley \& Sons, New York, 2010).

${ }^{34}$ W. H. Press, S. A. Teukolsky, W. T. Vetterling, and B. P. Flannery, Numerical Recipes in Fortran 77: The Art of Scientific Computing (Cambridge University Press, 1997).

${ }^{35}$ A. C. Albrecht, J. Chem. Phys. 34, 1476 (1961). 